\title{
The GALAH Survey: non-LTE departure coefficients for large spectroscopic surveys ${ }^{\star}$
}

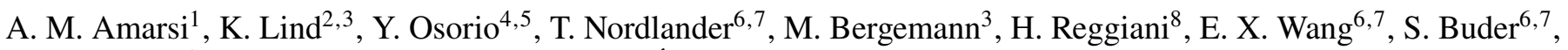 \\ M. Asplund ${ }^{6,7}$, P. S. Barklem ${ }^{1}$, A. Wehrhahn ${ }^{9}$, Á. Skúladóttir ${ }^{10,11}$, C. Kobayashi ${ }^{7,12}$, A. I. Karakas ${ }^{7,13}$, X. D. Gao ${ }^{3}$, \\ J. Bland-Hawthorn ${ }^{7,14}$, G. M. De Silva ${ }^{7,15}$, J. Kos ${ }^{16}$, G. F. Lewis ${ }^{14}$, S. L. Martel17,17, S. Sharma ${ }^{7,14}$, J. D. Simpson ${ }^{17}$, \\ D. B. Zucker ${ }^{18,19}$, K. Čotar ${ }^{16}$, J. Horner ${ }^{20}$, and the GALAH Collaboration
}

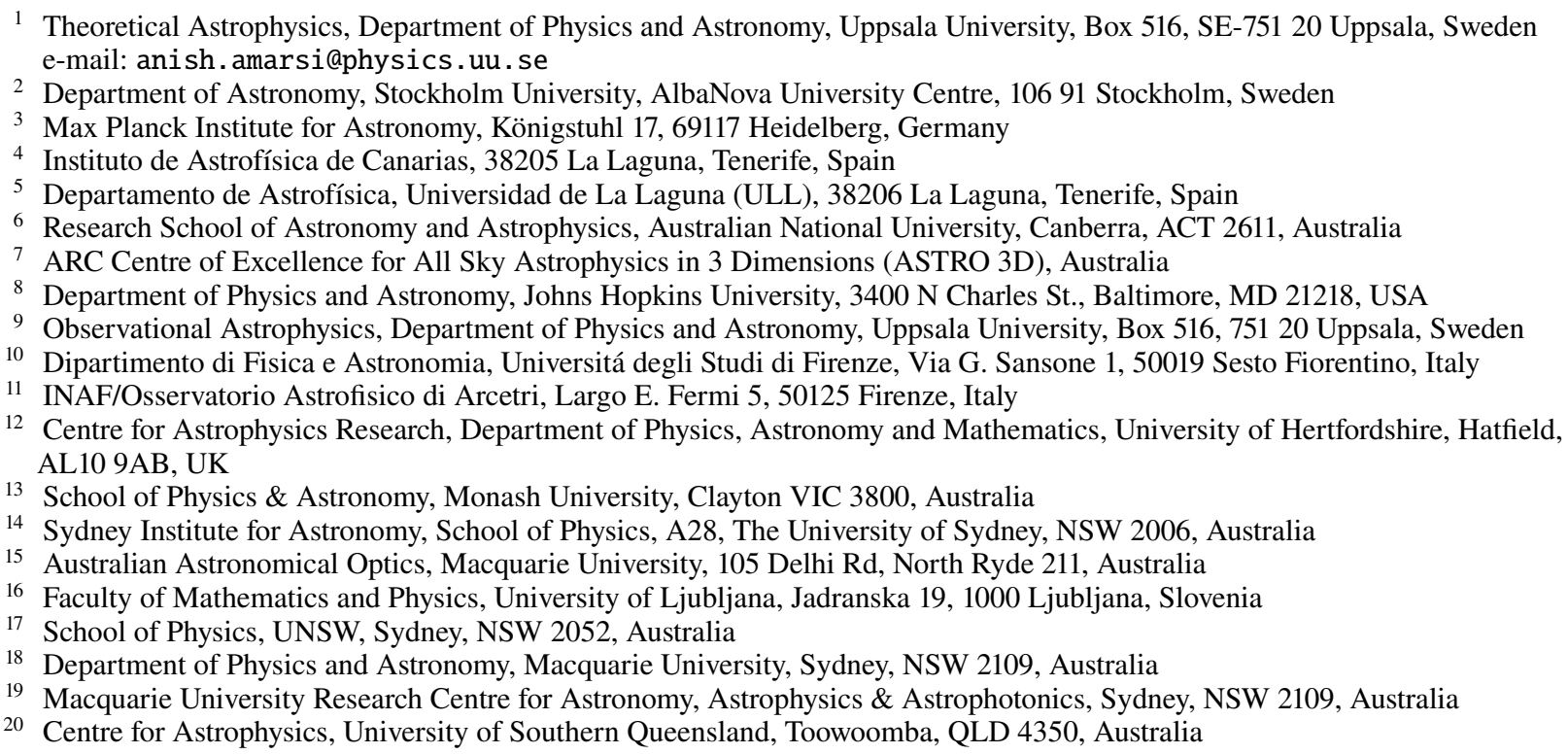

Received 12 June 2020 / Accepted 20 August 2020

\begin{abstract}
Massive sets of stellar spectroscopic observations are rapidly becoming available and these can be used to determine the chemical composition and evolution of the Galaxy with unprecedented precision. One of the major challenges in this endeavour involves constructing realistic models of stellar spectra with which to reliably determine stellar abundances. At present, large stellar surveys commonly use simplified models that assume that the stellar atmospheres are approximately in local thermodynamic equilibrium (LTE). To test and ultimately relax this assumption, we have performed non-LTE calculations for 13 different elements $(\mathrm{H}, \mathrm{Li}, \mathrm{C}, \mathrm{N}, \mathrm{O}, \mathrm{Na}, \mathrm{Mg}, \mathrm{Al}, \mathrm{Si}$, $\mathrm{K}, \mathrm{Ca}, \mathrm{Mn}$, and $\mathrm{Ba}$ ), using recent model atoms that have physically-motivated descriptions for the inelastic collisions with neutral hydrogen, across a grid of 3756 1D MARCS model atmospheres that spans $3000 \leq T_{\text {eff }} / \mathrm{K} \leq 8000,-0.5 \leq \log g / \mathrm{cm} \mathrm{s}^{-2} \leq 5.5$, and $-5 \leq[\mathrm{Fe} / \mathrm{H}] \leq 1$. We present the grids of departure coefficients that have been implemented into the GALAH DR3 analysis pipeline in order to complement the extant non-LTE grid for iron. We also present a detailed line-by-line re-analysis of 50126 stars from GALAH DR3. We found that relaxing LTE can change the abundances by between -0.7 dex and +0.2 dex for different lines and stars. Taking departures from LTE into account can reduce the dispersion in the $[\mathrm{A} / \mathrm{Fe}]$ versus $[\mathrm{Fe} / \mathrm{H}]$ plane by up to 0.1 dex, and it can remove spurious differences between the dwarfs and giants by up to 0.2 dex. The resulting abundance slopes can thus be qualitatively different in non-LTE, possibly with important implications for the chemical evolution of our Galaxy. The grids of departure coefficients are publicly available and can be implemented into LTE pipelines to make the most of observational data sets from large spectroscopic surveys.
\end{abstract}

Key words. atomic processes - radiative transfer - line: formation - stars: abundances - stars: atmospheres - Galaxy: abundances

\section{Introduction}

Stellar astronomy has entered a new era characterised by extremely large surveys of stars and their spectra. Massive

\footnotetext{
${ }^{\star}$ Grids of departure coefficients can be found online (Amarsi 2020) or by contacting the lead author directly.
}

studies of stellar parameters and elemental abundances, based on medium- or high-resolution spectra of around $10^{5}$ stars, are close to completion or have recently finished, including RAVE (Steinmetz et al. 2020; $R \sim 7500$ ), Gaia-ESO (Randich et al. 2013; $R \sim 20000$ ), APOGEE (Ahumada et al. 2020; $R \sim 22500$ ), and LAMOST (Liu et al. 2020; $R \sim 7500$ in the medium-resolution setting). The near future will see a jump 
in the number statistics. The planned WEAVE (Dalton et al. 2016) and 4MOST (de Jong et al. 2019) surveys in the northern and southern hemispheres, respectively, should obtain highresolution spectra for around $10^{7}$ stars, while the third Gaia data release (Gaia Collaboration 2018) will include mediumresolution spectra of around $10^{8}$ stars (Recio-Blanco et al. 2016). The ongoing Galactic Archaeology with HERMES (GALAH) survey falls somewhere in between these two groups, with the goal to observe $10^{6}$ stars at a relatively high resolution of $R \sim$ 28000 (De Silva et al. 2015).

Theoretical stellar spectra are used by all of these surveys in order to obtain stellar parameters and the elemental abundances. This is usually achieved by comparing the theoretical spectra against observations directly (García Pérez et al. 2016). Alternatively, they can also be used to train data-driven approaches (Ness et al. 2015; Ting et al. 2019) by directly using the theoretical spectra (Recio-Blanco et al. 2016) or gradient spectra (Xiang et al. 2019), or by first inferring precise stellar parameters and elemental abundances of a smaller sample of stars, which are then used as a training set (Buder et al. 2018).

The accuracy of these surveys depends on the reliability of their theoretical stellar spectra. This is particularly relevant for the determination of stellar elemental abundances. While extra constraints can be placed on effective temperatures, $T_{\text {eff }}$, and surface gravities, $\log g$, via interferometry, photometry, astrometry, and asteroseismology (Silva Aguirre et al. 2012; Karovicova et al. 2018, 2020), elemental abundances, in contrast, are most directly probed through absorption and emission lines in the stellar spectrum. It follows that deficiencies in the theoretical spectra have a direct impact on the reliability of the elemental abundance determinations.

A potential pitfall in classical spectroscopic analyses is the assumption that the stellar atmospheric matter satisfies local thermodynamic equilibrium (LTE; Mihalas \& Athay 1973). When calculating synthetic stellar spectra via radiative transfer post-processing of pre-computed model atmospheres (which themselves either assume strict LTE or adopt coherent isotropic continuum scattering; Gustafsson et al. 2008), this simplifying assumption allows one to describe the populations of the different energy levels of a given absorbing species using SahaBoltzmann statistics. This enables an immediate, analytical solution for the populations $n_{i}$ and $n_{j}$ of the lower and upper levels $i$ and $j$ for any given spectral line $i \leftrightarrow j$.

In reality, the radiation field is non-Planckian in stellar photospheres. Consequently, interactions between light and matter cause the latter to depart from LTE. A more general description of the level populations is given by the equations of statistical equilibrium (Rutten 2003):

$n_{i} \sum_{j}\left[R_{i j}+C_{i j}\right]=\sum_{j} n_{j}\left[R_{j i}+C_{j i}\right]$.

Or in other words, the net rate out of a level $i$ is set by the balance between all the outwards and inwards radiative $(R)$ and collisional $(C)$ transitions. Equation (1) is satisfied trivially in the stellar interior by virtue of the principle of detailed balance for LTE populations $n_{i}^{*}$ :

$n_{i}^{*} R_{i j} \equiv n_{j}^{*} R_{j i}$

$n_{i}^{*} C_{i j} \equiv n_{j}^{*} C_{j i}$.

However in the stellar photosphere, Eq. (2) no longer holds; nevertheless, since the particles have Maxwell-Boltzmann distributions to a good approximation (Hubeny \& Mihalas 2014),
Eq. (3) remains true for LTE populations $n_{i}^{*}$. Thus, the matter can only satisfy LTE, $n_{i}=n_{i}^{*}$, if the collisional rates dominate over the radiative rates; in general, this is not the case.

Solving for the statistical equilibrium is much more demanding than simply assuming LTE. Equation (1) indicates that all radiative and collisional transitions can affect the absorber populations and thus the spectral line. As well as being a much larger system of equations to solve, Eq. (1) must be iterated with a solution for the radiation field (Rybicki \& Hummer 1992), and convergence problems are often encountered. Moreover, nonLTE calculations require comprehensive sets of energy levels, and radiative and collisional transition probabilities; the final result is only as reliable as this input atomic data (Barklem 2016a). Therefore care is needed to calculate these data accurately, and then to consistently merge different data sources into what are referred to as model atoms. In contrast, when modelling a spectral line in LTE, the populations are fixed and one only needs to have the partition functions describing that particular chemical species and the parameters of that particular line.

One way to improve the accuracy of classical spectroscopic analyses without significantly increasing their cost is to apply pre-computed non-LTE solutions to them. A common approach is to use pre-computed absolute or differential abundance corrections $\Delta_{l}$ for a given spectral line $l^{1}$ :

$$
\begin{aligned}
\log \epsilon_{\mathrm{A} l}^{\text {Non-LTE }} & =\log \epsilon_{\mathrm{A} l}^{\mathrm{LTE}}+\Delta_{l}^{\mathrm{abs} .} \\
{[\mathrm{A} / \mathrm{H}]_{l}^{\text {Non-LTE }} } & =[\mathrm{A} / \mathrm{H}]_{l}^{\mathrm{LTE}}+\Delta_{l}^{\text {diff. }} .
\end{aligned}
$$

Vast grids of line-by-line abundance corrections for many different atomic species already exist in the literature (e.g. Bergemann et al. 2012; Lind et al. 2012; Korotin et al. 2015; Mashonkina et al. 2016; Osorio \& Barklem 2016; Amarsi et al. 2019b), that can readily be adopted and applied by the stellar spectroscopy community.

The line-by-line abundance corrections approach (Eq. (4)) can become prohibitively complicated when a large number of spectral lines need to be studied simultaneously. It is simpler to instead apply non-LTE corrections to the level populations, because the number of relevant levels that would need to be considered roughly scales with the square root of the corresponding number of relevant spectral lines. This can be accomplished using pre-computed grids of non-LTE departure coefficients $\beta_{i}$, for a given energy level $i$ :

$\beta_{i} \equiv \frac{n_{i}}{n_{i}^{*}}$

This approach requires some extra initial effort, because LTE spectrum synthesis codes need to be modified to read and manipulate the grids of departure coefficients. Nevertheless, grids of departure coefficients are desirable for large spectroscopic surveys, which are typically based on full-spectrum analyses.

Here, we present publicly-available grids of departure coefficients for 13 different elements: $\mathrm{H}, \mathrm{Li}, \mathrm{C}, \mathrm{N}, \mathrm{O}, \mathrm{Na}, \mathrm{Mg}, \mathrm{Al}, \mathrm{Si}$, $\mathrm{K}, \mathrm{Ca}, \mathrm{Mn}$, and $\mathrm{Ba}$. For many of these elements, this is the most extensive set of non-LTE calculations: they cover 3756 1D model atmospheres that span the HR diagram from M-dwarfs, up the main sequence past the turn-off, to the tip of the red giant branch, with $-5 \leq[\mathrm{Fe} / \mathrm{H}] \leq 1$. This is also the first time that consistently

\footnotetext{
1 The absolute abundance of element $\mathrm{A}$ is defined as $\log \epsilon_{\mathrm{A}} \equiv$ $\log _{10}\left(N_{\mathrm{A}} / N_{\mathrm{H}}\right)+12$, where $N_{\mathrm{A}}$ and $N_{\mathrm{H}}$ are the number of nuclei of element $A$ and of hydrogen. Abundance ratios differential to the Sun are defined as $[\mathrm{A} / \mathrm{H}] \equiv \log \epsilon_{\mathrm{A}}-\log \epsilon_{\mathrm{A} \odot}$ and $[\mathrm{A} / \mathrm{Fe}] \equiv[\mathrm{A} / \mathrm{H}]-[\mathrm{Fe} / \mathrm{H}]$.
} 
computed grids of departure coefficients for multiple elements have been released in the literature.

In Sect. 2 we describe the non-LTE radiative transfer calculations, and the implementation of the resulting grids of departure coefficients into the GALAH analysis pipeline. In Sect. 3 we present a line-by-line re-analysis of 55159 spectra corresponding to 50126 FGK-type field stars from GALAH DR3, and in Sect. 4 use these results to discuss the impact of the departure coefficients in practice. In Sect. 5 we briefly discuss the implication of our non-LTE abundance analysis in the context of Galactic chemical evolution, and in Sect. 6 we summarise and make some concluding remarks about the outlook of quantitative non-LTE stellar spectroscopy.

\section{Calculation of departure coefficients}

\subsection{Model atoms}

We list the 13 elements considered in this work and give the sizes of the corresponding model atoms in Table 1. Full details about the physics included in each of the models can be found in the original papers, references to which are also provided in Table 1. Here we provide a very brief overview of the models.

The adopted model atoms were all constructed recently, the oldest being that for sodium (Lind et al. 2011). Minor changes were made to some of the models compared to what was presented in the original papers, in order to reduce the computational cost. In particular, for carbon, nitrogen, magnesium, and calcium, all fine structure levels were collapsed. This has previously been verified for carbon (Amarsi et al. 2019a) and oxygen (Amarsi et al. 2018a) to have only a small impact on the predicted departures from LTE: typically of the order 0.01 dex in terms of abundances.

For completeness, the model atom for iron is also listed in Table 1. Iron is treated in non-LTE in GALAH DR2 (Buder et al. 2018) and DR3 (Buder et al., in prep.). However the departure coefficients originate from an older set of calculations described in Amarsi et al. (2016b). They are not presented and discussed in the present study.

Inelastic collisions with neutral hydrogen have historically been one of the largest sources of uncertainty in non-LTE models (Asplund 2005). Until recently, if such processes were not neglected completely, the classical Drawin recipe would usually be adopted. This recipe is based on the Thomson cross-section for ionisation by electron collisions (Thomson 1912), as modified for ionisation by atomic collisions by Drawin $(1969,1968)$, and later extended by Steenbock \& Holweger (1984) and Lambert (1993) to also cover excitation. However, the Drawin recipe does not reflect the actual physics of the low-energy collisions occurring in stellar atmospheres (Barklem et al. 2011; Barklem 2016a; Belyaev \& Yakovleva 2017). To attempt to correct for this, usually a single fudge factor $S_{\mathrm{H}}$ is calibrated and applied to the Drawin rate coefficients (Allende Prieto et al. 2004; Steffen et al. 2015). This approach is not guaranteed to improve the reliability of the models: apart from introducing more free parameters into the spectroscopic analysis, this does not take into account that the errors in the Drawin recipe vary depending on the stellar parameters, and are transition-dependent. The Drawin recipe is also unable to describe charge transfer processes $(\mathrm{A}+\mathrm{H} \leftrightarrow$ $\mathrm{A}^{+}+\mathrm{H}^{-}$), which are of astrophysical importance (Barklem et al. 2011).

To avoid the inherent uncertainties of the Drawin recipe, all of the adopted model atoms use alternative, physically-motivated descriptions for the inelastic collisions with neutral hydrogen.
Table 1. Number of levels, lines, and continua, and the original references of the adopted model atoms.

\begin{tabular}{|c|c|c|c|c|c|}
\hline Element & Species & \# levels & \# lines & \# continua & Ref. \\
\hline \multirow{2}{*}{$\mathrm{H}$} & $\mathrm{H} \mathrm{I}$ & 20 & 190 & 20 & \multirow{2}{*}{1} \\
\hline & H II & 1 & - & - & \\
\hline \multirow{2}{*}{$\mathrm{Li}$} & $\mathrm{Li}$ I & 20 & 113 & 20 & \multirow{2}{*}{2,3} \\
\hline & Li II & 1 & - & - & \\
\hline \multirow{2}{*}{$\mathrm{C}$} & $\mathrm{C}_{\mathrm{I}}$ & 46 & 343 & 45 & \multirow{2}{*}{4} \\
\hline & C II & 1 & - & - & \\
\hline \multirow{2}{*}{$\mathrm{N}$} & $\mathrm{N}_{\mathrm{I}}$ & 31 & 174 & 32 & \multirow{2}{*}{5} \\
\hline & $\mathrm{N}$ II & 2 & - & - & \\
\hline \multirow{2}{*}{$\mathrm{O}$} & $\mathrm{O}_{\mathrm{I}}$ & 47 & 322 & 47 & \multirow{2}{*}{6} \\
\hline & O II & 3 & - & - & \\
\hline \multirow{2}{*}{$\mathrm{Na}$} & $\mathrm{Na} \mathrm{I}$ & 22 & 166 & 22 & \multirow{2}{*}{7} \\
\hline & $\mathrm{Na}$ II & 1 & - & - & \\
\hline \multirow{3}{*}{$\mathrm{Mg}$} & $\mathrm{Mg} I$ & 96 & 721 & 96 & \multirow{3}{*}{8} \\
\hline & Mg II & 29 & 161 & 29 & \\
\hline & Mg III & 1 & - & - & \\
\hline \multirow{2}{*}{$\mathrm{Al}$} & $\mathrm{Al} \mathrm{I}$ & 42 & 135 & 33 & \multirow{2}{*}{9} \\
\hline & Al II & 2 & - & - & \\
\hline \multirow{2}{*}{$\mathrm{Si}$} & Si I & 56 & 634 & 56 & \multirow{2}{*}{10} \\
\hline & Si II & 1 & - & - & \\
\hline \multirow{2}{*}{$\mathrm{K}$} & $\mathrm{K}_{\mathrm{I}}$ & 133 & 250 & 133 & \multirow{2}{*}{11} \\
\hline & K II & 1 & - & - & \\
\hline \multirow{3}{*}{$\mathrm{Ca}$} & $\mathrm{Ca}$ I & 67 & 937 & 67 & \multirow{3}{*}{12} \\
\hline & Ca II & 24 & 89 & 24 & \\
\hline & Ca III & 1 & - & - & \\
\hline \multirow{3}{*}{$\mathrm{Mn}$} & Mn I & 198 & 1645 & 198 & \multirow{3}{*}{13} \\
\hline & Mn II & 82 & 44 & - & \\
\hline & Mn III & 1 & - & - & \\
\hline \multirow{3}{*}{$\mathrm{Fe}$} & $\mathrm{Fe} I$ & 421 & 3923 & 48 & \multirow{3}{*}{14,15} \\
\hline & Fe II & 41 & 77 & - & \\
\hline & Fe III & 1 & - & - & \\
\hline \multirow{3}{*}{$\mathrm{Ba}$} & $\mathrm{Ba} \mathrm{I}$ & 8 & - & 8 & \multirow{3}{*}{16} \\
\hline & Ba II & 102 & 284 & 102 & \\
\hline & Ba III & 1 & - & - & \\
\hline
\end{tabular}

Notes. A non-LTE grid for iron (highlighted) is also adopted in GALAH; details of those calculations can be found in Amarsi et al. (2016b).

References. (1) Amarsi et al. (2018b); (2) Lind et al. (2013); (3) Wang et al. (in prep.); (4) Amarsi et al. (2019a); (5) Amarsi et al. (2020); (6) Amarsi et al. (2018a); (7) Lind et al. (2011); (8) Osorio et al. (2015); (9) Nordlander \& Lind (2017); (10) Amarsi \& Asplund (2017); (11) Reggiani et al. (2019); (12) Osorio et al. (2019); (13) Bergemann et al. (2019); (14) Amarsi et al. (2016b); (15) Lind et al. (2017); (16) Gallagher et al. (2020).

For transitions involving low- and intermediate-lying levels of lithium (Belyaev \& Barklem 2003; Barklem et al. 2003), sodium (Belyaev et al. 2010; Barklem et al. 2010), and magnesium (Belyaev et al. 2012; Barklem et al. 2012), cross-sections based on full quantum chemistry calculations were used. For the other species, calculations based on asymptotic methods were used (Belyaev 2013; Barklem 2016b). In addition, with the exception of barium (Gallagher et al. 2020), transitions involving Rydberg 

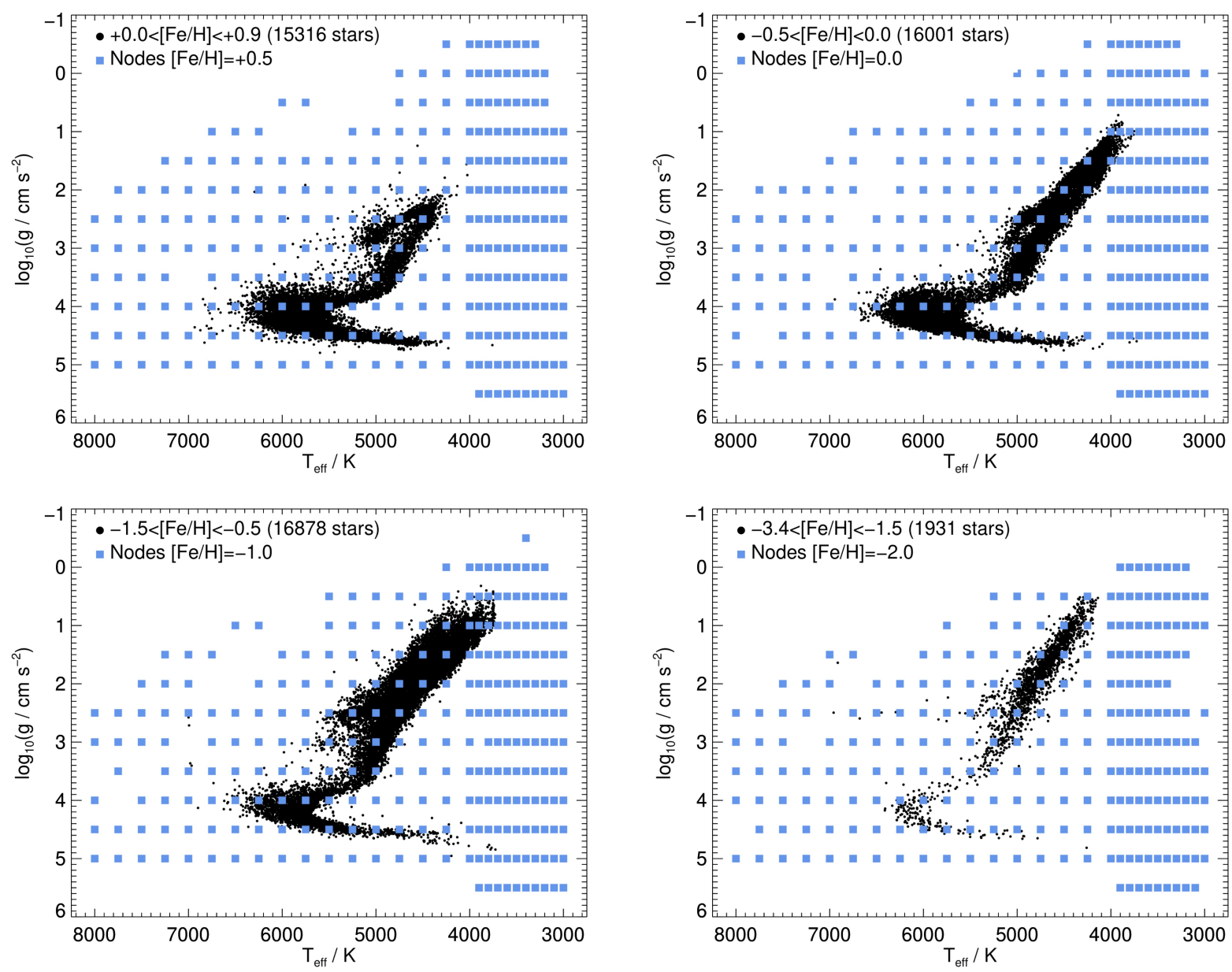

Fig. 1. Grid nodes (surface gravities and effective temperatures) at which the non-LTE radiative transfer calculations were carried out. The missing nodes are where standard MARCS model atmospheres do not presently exist, owing to convergence issues (see Sect. 5.4 of Gustafsson et al. 2008). Also shown in black are the parameters of the 50126 stars drawn from GALAH DR3, studied in this work.

levels were described using the free electron method (Kaulakys 1985, 1986, 1991).

\subsection{Model atmospheres}

The departure coefficients were calculated for a grid of 1D MARCS model atmospheres (Gustafsson et al. 2008), available from the online repository ${ }^{2}$. The models specify the gas temperature, density, and other thermodynamic quantities, on 56 depth points. In this work, these quantities were not re-sampled and interpolated onto a new depth scale. This means that the departure coefficients presented here can readily be adopted and used consistently with the standard MARCS models.

We illustrate the extent of the grid of model atmospheres in Figs. 1 and 2. The 3756 different models are labelled by $T_{\text {eff }}, \log g$, and $[\mathrm{Fe} / \mathrm{H}]$, and have the standard MARCS chemical compositions: namely, they adopt the solar chemical compositions of Grevesse et al. (2007), scaled with $[\mathrm{Fe} / \mathrm{H}]$, and with an enhancement to $\alpha$ elements of $+0.1,+0.2,+0.3$, and +0.4 for $[\mathrm{Fe} / \mathrm{H}]=-0.25,-0.5,-0.75$, and $[\mathrm{Fe} / \mathrm{H}] \leq-1.0$ respectively. Plane-parallel models with $\xi_{\text {mic }}=1.0 \mathrm{~km} \mathrm{~s}^{-1}$ were adopted for dwarfs $\left(\log g / \mathrm{cm} \mathrm{s}^{-2}>3.5\right.$ in Fig. 1), and sphericallysymmetric models with $\xi_{\text {mic }}=2.0 \mathrm{~km} \mathrm{~s}^{-1}$ were adopted for giants $\left(\log g / \mathrm{cm} \mathrm{s}^{-2} \leq 3.5\right.$ in Fig. 1).

\footnotetext{
2 marcs.astro.uu.se
}

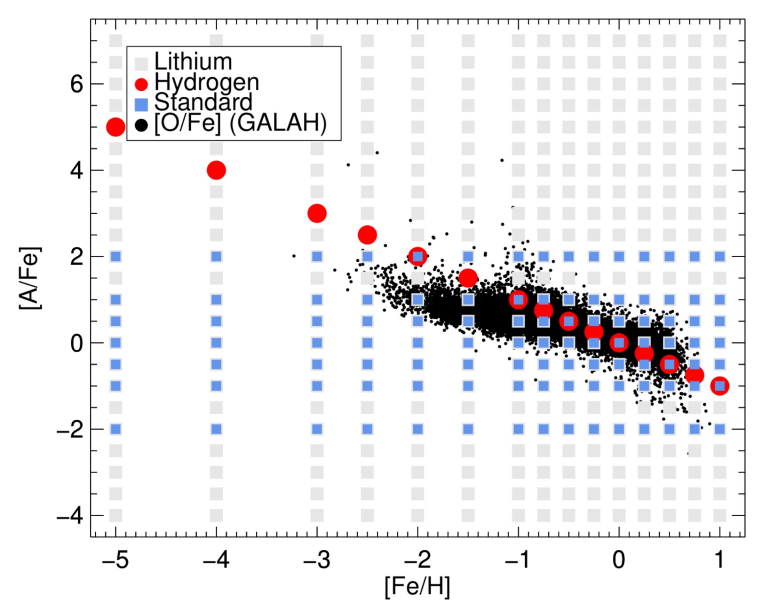

Fig. 2. Grid nodes (abundance ratios and metallicities) at which the nonLTE radiative transfer calculations were carried out. The calculations for hydrogen and lithium are specially noted. Also shown in black are $[\mathrm{O} / \mathrm{Fe}]$ for the sample of stars discussed in the present study.

\subsection{Non-LTE radiative transfer}

The departure coefficients were calculated using the MPIparallelised non-LTE radiative transfer code Balder (Amarsi et al. 2018b), which is our modified version of Multi3D 
(Leenaarts \& Carlsson 2009). The code takes as input a model atom (Sect. 2.1) and a model atmosphere (Sect. 2.2). Balder uses the algorithm described in Sect. 2.4 of Rybicki \& Hummer (1992) to solve the equations of statistical equilibrium Eq. (1), together with the radiative transfer equation on short characteristics (Ibgui et al. 2013); the system of equations are closed by enforcing population conservation. Acceleration of convergence was achieved using the generalised conjugate residual algorithm (GCR; see the Appendix of Kao \& Auer 1990, and Saad 2003).

The code Blue (Amarsi et al. 2016b) was used within Balder to determine the equation-of-state (assumed to be in LTE) and the background opacities for around $10^{6}$ lines from atomic and ionic species and $10^{8}$ lines from molecular species. The bound-free (continuous) opacities were calculated on-thefly, whilst the bound-bound (line) opacities were pre-computed for different temperature-density-metallicity combinations, and interpolated at runtime. Care was taken not to double-count opacities already included in the model atoms. Partition functions and dissociation constants were drawn from Barklem \& Collet (2016), and details of which background bound-bound and bound-free transitions were included can be found in Sect. 2.1.2 of Amarsi et al. (2016b).

The non-LTE radiative transfer calculations were performed independently for different elements, for a variety of elemental abundances, as we illustrate in Fig. 2. Generally, for a given element A, and for a given model atmosphere with chemical composition labelled by $[\mathrm{Fe} / \mathrm{H}]$, calculations were performed for different abundances $-2 \leq[\mathrm{A} / \mathrm{Fe}] \leq+2$, keeping the abundances of all other elements fixed (employing the trace element approximation), but with the equation-of-state recomputed each time. There are some exceptions, however: for hydrogen, the calculations were kept strictly consistent with the composition of the model atmosphere; and for lithium, calculations were performed for $-4 \leq[\mathrm{Li} / \mathrm{Fe}] \leq+7$.

The departure coefficients are very weakly sensitive to the input microturbulence parameter. A depth-independent microturbulence was adopted, the value of which was chosen to be consistent with that of the model atmosphere (Sect. 2.2): namely, $\xi_{\text {mic }}=1.0 \mathrm{~km} \mathrm{~s}^{-1}$ for dwarfs $\left(\log g / \mathrm{cm} \mathrm{s}^{-2}>3.5\right.$ in Fig. 1), and $\xi_{\text {mic }}=2.0 \mathrm{~km} \mathrm{~s}^{-1}$ for giants (log $g / \mathrm{cm} \mathrm{s}^{-2} \leq 3.5$ in Fig. 1). Although the microturbulence is fixed to these values for the calculation of the grid of departure coefficients, the microturbulence is varied in GALAH DR3 and in the re-analysis we present in Sect. 3, using the empirical formula described in Buder et al. (2018).

The non-LTE calculations were assumed to have converged once the monochromatic emergent intensities changed by less than $0.01 \%$ between successive iterations. Out of the total number of individual non-LTE runs (383036), only a small number of them (2089) did not reach this convergence criterion. Various types of convergence problems affected different runs, depending on the element and on the region of the parameter space. Given the scope of this project these problems could not be addressed individually. Nevertheless, we found that typically the departure coefficients of the runs that did not formally converge looked physically reasonable, after comparing against those from converged runs having similar stellar parameters. We include them in the data set, but cannot preclude the possibility of some unphysical behaviour in some regions of the parameter space.

\subsection{Background scattering}

Owing to limited computational resources at the time, the results presented here and adopted into GALAH DR3 are based on an initial set of non-LTE calculations wherein all background species are assumed to strictly satisfy LTE. In other words, the background source function is equal to the Planck function:

$S_{v}^{\text {background }}=B_{v}$.

Separate calculations were later carried out wherein scattering for lines from background atomic and ionic species were included, assuming it to be isotropic and coherent:

$S_{v}^{\text {background }}=\epsilon_{v} B_{v}+\left(1-\epsilon_{v}\right) J_{v}$.

Here, $J_{v}$ is the mean radiation field, and $\epsilon_{v}$ is the photon destruction probability. For background lines, the latter quantity were estimated using Eq. (3.98) of Rutten (2003), which is valid for two level atoms:

$\epsilon_{v ; j i}=\frac{C_{j i}}{C_{j i}+A_{j i}+B_{j i} B_{v}}$.

When estimating the photon destruction probabilities, electron collisions were assumed to dominate, and these rates were estimated using Eq. (22) of van Regemorter (1962). In addition, Thomson scattering of free electrons and Rayleigh scattering of atomic hydrogen in the red wing of the Lyman series were also included, the latter following Lee \& Kim (2004).

The two sets of calculations allow us to quantify the impact of background scattering on GALAH DR3 abundances. In general, the effects on most of the GALAH lines are small, across the entire parameter space. The effects on the Li I, O I, Na I, Al I, $\mathrm{Si}$ I, and $\mathrm{KI}$ lines in Table 2 are at most $0.01 \mathrm{dex}$, for dwarfs and giants. The effects are more severe for the $\mathrm{CI}, \mathrm{Mg}$ I, Ca I, and Mn I lines. For these species, the effects can be important for giants $\left(\log \mathrm{g} / \mathrm{cm} \mathrm{s}^{-2} \leq 3.5\right)$, towards lower metallicities. Our tests imply that the non-LTE GALAH results for carbon may be slightly overestimated, and the non-LTE GALAH results for magnesium, calcium, and manganese abundances may be slightly underestimated, in metal-poor giants by $0.01-0.05$ dex; and in extreme cases (mainly in the parameter space around $T_{\text {eff }} \approx 5000 \mathrm{~K}, \log g / \mathrm{cm} \mathrm{s}^{-2} \approx 2$, and $[\mathrm{Fe} / \mathrm{H}] \lesssim-2$ ) by up to 0.1 dex. Nevertheless, metal-poor giants are only a small fraction of the GALAH sample (Fig. 1), hence treating the background in LTE is well justified for the vast majority of stars, including for the $\mathrm{CI}, \mathrm{Mg}$ I, Ca I, and Mn I lines considered in this study.

\subsection{Implementation of departure coefficients into LTE spectrum synthesis codes}

The grids of departure coefficients can be found online (Amarsi 2020) or by contacting the lead author directly. These departure coefficients include background scattering as described in Sect. 2.4; the departure coefficients that do not include background scattering, which were used in GALAH DR3, are also available upon request. The departure coefficients, defined by Eq. (6), are given for different energy levels $i$ of the different elements $\mathrm{A}$ in Table 1, at each depth point $z$ in the different model atmospheres labelled by $T_{\text {eff }}, \log g$, and $[\mathrm{Fe} / \mathrm{H}]$ in Sect. 2.2:

$\beta_{i} \equiv \beta_{i(\mathrm{~A})}\left([\mathrm{A} / \mathrm{Fe}], T_{\mathrm{eff}}, \log g,[\mathrm{Fe} / \mathrm{H}], z\right)$.

The levels in each of the model atoms were matched to the species, electron configurations, spectroscopic terms, and total angular momentum quantum numbers $J$ listed in the NIST Atomic Spectra Database version 5.7 (Kramida et al. 2012). If $J$ was not resolved in the model atom, the fine structure levels were 
Table 2. Lines used in the reanalysis of GALAH DR3 stars.

\begin{tabular}{|c|c|c|c|c|c|c|c|c|c|c|c|c|}
\hline \multirow{2}{*}{ Spec. } & \multirow{2}{*}{$\lambda_{\text {air }} / \mathrm{nm}$} & \multirow{2}{*}{$\chi_{\text {low }} / \mathrm{eV}$} & \multirow{2}{*}{$\log g f$} & \multirow{2}{*}{ Ref. } & \multirow{2}{*}{$\log \epsilon_{\odot}^{\text {Non-LTE }}$} & \multirow{2}{*}{$\log \epsilon_{\odot}^{\mathrm{LTE}}$} & \multicolumn{3}{|c|}{$-2<[\mathrm{Fe} / \mathrm{H}]<-1$} & \multicolumn{3}{|c|}{$0<[\mathrm{Fe} / \mathrm{H}]<1$} \\
\hline & & & & & & & K V & $\mathrm{FV}$ & K III & K V & F V & K III \\
\hline $\mathrm{Li}$ I & $\begin{array}{l}670.776 \\
670.791\end{array}$ & $\begin{array}{l}0.000 \\
0.000\end{array}$ & $\begin{array}{l}-0.002 \\
-0.303\end{array}$ & 1 & $(1.05)$ & $(1.05)$ & -0.05 & -0.10 & -0.02 & -0.02 & -0.07 & +0.02 \\
\hline$\overline{C_{I}}$ & 658.761 & 8.537 & -1.021 & 2 & 8.43 & 8.43 & & -0.02 & +0.00 & +0.00 & -0.01 & +0.00 \\
\hline$\overline{\mathrm{OI}}$ & 777.194 & 9.146 & +0.369 & 3 & 8.79 & 8.99 & -0.06 & -0.26 & -0.12 & -0.08 & -0.52 & -0.12 \\
\hline O I & 777.417 & 9.146 & +0.223 & 3 & 8.80 & 8.98 & -0.04 & -0.22 & -0.12 & -0.07 & -0.48 & -0.13 \\
\hline O I & 777.539 & 9.146 & +0.002 & 3 & 8.79 & 8.94 & -0.05 & -0.19 & -0.12 & -0.08 & -0.42 & -0.07 \\
\hline $\mathrm{Na} \mathrm{I}$ & 568.263 & 2.102 & -0.706 & 4 & 6.04 & 6.19 & -0.10 & -0.12 & -0.12 & -0.13 & -0.17 & -0.23 \\
\hline $\mathrm{Na} \mathrm{I}$ & 568.821 & 2.104 & -0.404 & 4 & 6.05 & 6.24 & -0.10 & -0.14 & -0.17 & -0.14 & -0.23 & -0.19 \\
\hline $\mathrm{Mg} \mathrm{I}$ & 571.109 & 4.346 & -1.724 & 5 & 7.59 & 7.63 & -0.03 & +0.03 & -0.11 & -0.06 & -0.05 & -0.18 \\
\hline$\overline{\mathrm{Al} \mathrm{I}}$ & 669.867 & 3.143 & -1.870 & 6 & 6.39 & 6.41 & -0.03 & & -0.09 & -0.03 & -0.01 & -0.10 \\
\hline $\mathrm{Al} \mathrm{I}$ & 783.531 & 4.022 & -0.689 & 6 & 6.41 & 6.43 & -0.03 & & -0.07 & -0.03 & -0.02 & -0.13 \\
\hline$\overline{\mathrm{Si} I}$ & 568.448 & 4.954 & -1.553 & 7,8 & 7.39 & 7.42 & -0.01 & +0.00 & -0.06 & -0.02 & -0.03 & -0.08 \\
\hline Si I & 569.043 & 4.930 & -1.773 & 7,8 & 7.43 & 7.44 & -0.01 & +0.00 & -0.05 & -0.01 & -0.02 & -0.06 \\
\hline Si I & 570.110 & 4.930 & -1.953 & 7,8 & 7.49 & 7.50 & -0.01 & & -0.04 & -0.01 & -0.02 & -0.03 \\
\hline Si I & 577.215 & 5.082 & -1.653 & 7,8 & 7.50 & 7.52 & -0.01 & -0.01 & -0.04 & -0.01 & -0.02 & -0.07 \\
\hline Si I & 579.307 & 4.930 & -1.963 & 7,8 & 7.48 & 7.50 & -0.01 & -0.01 & -0.04 & -0.01 & -0.02 & -0.11 \\
\hline Si I & 768.027 & 5.863 & -0.590 & 7,8 & 7.57 & 7.61 & -0.02 & -0.01 & -0.11 & -0.04 & -0.06 & -0.25 \\
\hline $\mathrm{K}_{\mathrm{I}}$ & 769.896 & 0.000 & -0.178 & 9 & 5.05 & 5.49 & -0.28 & -0.28 & -0.66 & -0.21 & -0.62 & -0.30 \\
\hline$\overline{\mathrm{Ca} \mathrm{I}}$ & 586.756 & 2.933 & -1.570 & 10 & 6.32 & 6.32 & -0.01 & & -0.01 & -0.01 & -0.01 & -0.03 \\
\hline $\mathrm{Ca} \mathrm{I}$ & 649.965 & 2.523 & -0.818 & 11 & 6.27 & 6.31 & -0.06 & +0.00 & -0.01 & -0.05 & +0.01 & +0.02 \\
\hline Mn I & 475.404 & 2.282 & -0.080 & 12 & 5.29 & 5.28 & +0.06 & +0.15 & +0.10 & +0.00 & +0.00 & +0.02 \\
\hline Mn I & 476.151 & 2.953 & -0.274 & 12 & 5.36 & 5.34 & +0.05 & +0.12 & +0.12 & +0.01 & +0.03 & +0.02 \\
\hline Ba II & 585.367 & 0.604 & -0.907 & 13 & 2.13 & 2.22 & -0.01 & -0.04 & -0.12 & -0.04 & -0.14 & -0.03 \\
\hline Ba II & 649.689 & 0.604 & -0.407 & 13 & 2.23 & 2.42 & -0.07 & -0.12 & -0.18 & -0.10 & -0.29 & -0.07 \\
\hline
\end{tabular}

Notes. The two components of the Li I doublet were fit simultaneously. Also shown are the solar abundances inferred via the solar twins in the sample; the value for the $\mathrm{Li}$ I doublet in brackets was adopted from the literature (Sect. 3.2). The final columns show the typical absolute abundance corrections $\left(\log \epsilon^{\mathrm{Non}-\mathrm{LTE}}-\log \epsilon^{\mathrm{LTE}}\right)$ for each line: median results are shown in $\left(T_{\mathrm{eff}} / K \pm 250, \log g / \mathrm{cm} \mathrm{s}^{-2} \pm 0.5\right)$ regions corresponding to spectral and luminosity classes K V $(5000,4.5), \mathrm{F}$ V $(6500,4.0)$, and K III $(4250,2.0)$. The most negative and positive corrections for a given line are highlighted, and no results are shown for where the lines were too weak to be detected.

References. (1) Yan et al. (1998); (2) Hibbert et al. (1993); (3) Hibbert et al. (1991); (4) Froese Fischer et al. (2006); (5) Chang \& Tang (1990); (6) Kelleher \& Podobedova (2008); (7) Garz (1973); (8) O'Brian \& Lawler (1991); (9) Trubko et al. (2017); (10) Smith (1988); (11) Smith \& Raggett (1981); (12) Den Hartog et al. (2011); (13) Davidson et al. (1992).

assigned identical departure coefficients. Levels in NIST that are missing in the model atoms were assigned departure coefficients corresponding to those of the levels nearest in energy, within the same spin system, with zero-order extrapolation for most ions (the exceptions being the ionisation stages that do not extend to Rydberg states in the model atoms, including Ba I and Mn II).

In addition to ASCII format, grids are provided that are compatible with the spectrum synthesis code Spectroscopy Made Easy (SME; Valenti \& Piskunov 1996), and the python version PySME $^{3}$ (Wehrhahn et al., in prep.). Details about the way in which the departure coefficients are used to generate non-LTE synthetic spectra can be found in Sect. 3 of Piskunov \& Valenti (2017) and in the online documentation ${ }^{4}$. Given a line list, SME or PySME looks for the departure coefficients of the lower and upper levels of all spectral lines by matching the species, electron configurations, spectroscopic terms, and total angular momentum quantum numbers $J$ specified in the grids. It is therefore

\footnotetext{
3 https://github.com/AWehrhahn/SME

4 https://pysme-astro.readthedocs.io
}

important that the user ensures the format of these labels in their line list is consistent with the format used in the departure coefficient grids.

The grids of departure coefficients (without background scattering) have been implemented into the GALAH analysis pipeline, that is based on SME as described in Buder et al. (2018). The grids have been used for determining elemental abundances in GALAH DR3 (Buder et al., in prep.). We have also used them in the line-by-line re-analysis of GALAH DR3 stars, as we discuss in Sect. 3 below.

\section{Re-analysis of GALAH DR3 stars}

\subsection{Stellar sample}

The full GALAH DR3 stellar sample (Buder et al., in prep.) includes over 600000 spectra corresponding to over 500000 stars. The GALAH DR3 data products include $T_{\text {eff }}, \log g$, a first estimate of the iron abundance $[\mathrm{Fe} / \mathrm{H}]_{\mathrm{atmo}}$, microturbulence $\xi_{\text {mic }}$, and a broadening parameter $v_{\text {broad }}$ that reflects the combined 
effects of stellar rotation and macroturbulence. These stellar parameters were determined simultaneously, with hydrogen modelled in non-LTE using the departure coefficients presented in this work, and iron modelled in non-LTE using the departure coefficients presented in an older, but similar, set of calculations (Amarsi et al. 2016b). Also provided are elemental abundance ratios $[\mathrm{A} / \mathrm{Fe}]$, that were determined in a second step with the stellar parameters fixed. In particular, the non-LTE abundances were determined using the departure coefficients presented in this work (albeit without background scattering; Sect. 2.4).

To show the impact of the departure coefficients, a reanalysis of GALAH DR3 was carried out on 55159 spectra corresponding to 50126 stars. These spectra were selected out of GALAH DR3 such that they correspond to field stars, of spectral type FGK, and have the lowest uncertainties in the stellar parameters as stipulated by the GALAH analysis pipeline (Buder et al. 2018). The stellar parameters $\left(T_{\text {eff }}, \log g,[\mathrm{Fe} / \mathrm{H}]\right)$ were fixed to the values provided with GALAH DR3, however the abundances were rederived using reduced GALAH spectra (Kos et al. 2017) and the GALAH analysis pipeline, modifying the spectral masks so that separate abundances were derived from the different lines (Sect. 3.2). This re-analysis was necessary because LTE abundances are not provided with GALAH DR3, for the elements listed in Table 1. Moreover, for the elements considered here, GALAH DR3 does not include information about the line-byline abundance dispersion, because the elemental abundances were determined from simultaneous fits of all of the available lines of that element.

\subsection{Line list and solar abundances}

In Table 2 we list the lines used in the re-analysis of 50126 stars from GALAH DR3. All of the elements in Table 1 were considered, except nitrogen, due to the lack of suitable lines within the GALAH spectral range. The line list has a large overlap with that of GALAH DR3 (Buder et al., in prep.).

We also list the adopted line-by-line absolute solar elemental abundances $\log \epsilon$ in Table 2 . These were determined from the mean result of 281 solar twins present in the sample: stars with $T_{\text {eff }}$ within $100 \mathrm{~K}, \log g$ within $0.1 \mathrm{dex}$, and $[\mathrm{Fe} / \mathrm{H}]$ within 0.1 dex from the Sun (Nissen \& Gustafsson 2018). The number of solar twins is sufficient to average out the intrinsic dispersions that are due to their different ages (Nissen 2015; Spina et al. 2016; Bedell et al. 2018), so the zero points in Sect. 3 should closely reflect the actual results for the Sun. For lithium the standard value of $\log \epsilon_{\mathrm{Li}}=1.05$ (Asplund et al. 2009) is listed in Table 2, however the stellar lithium abundances discussed in this paper are given in the absolute sense, as per convention.

Subsequent sections are based on the abundance ratios $[\mathrm{A} / \mathrm{H}]$ or $[\mathrm{A} / \mathrm{Fe}]$ relative to the Sun; these were determined differentially on a line-by-line basis using the solar elemental abundances given in Table 2, prior to averaging over the lines. Owing to the large number of solar twins in the sample, this method of normalising the elemental abundances precisely puts the different spectral lines onto the same scale. It thus leads to a significant cancellation of errors originating from the adopted oscillator strengths, systematic offsets in the continuum placements, and the neglected effects of stellar surface convection. In the LTE results, this also cancels some of the errors arising from the neglected non-LTE effects, as we show in Sect. 4.1.

\subsection{Stellar elemental abundances}

We show the difference between the inferred LTE and non-LTE elemental abundances (the differential abundance corrections;

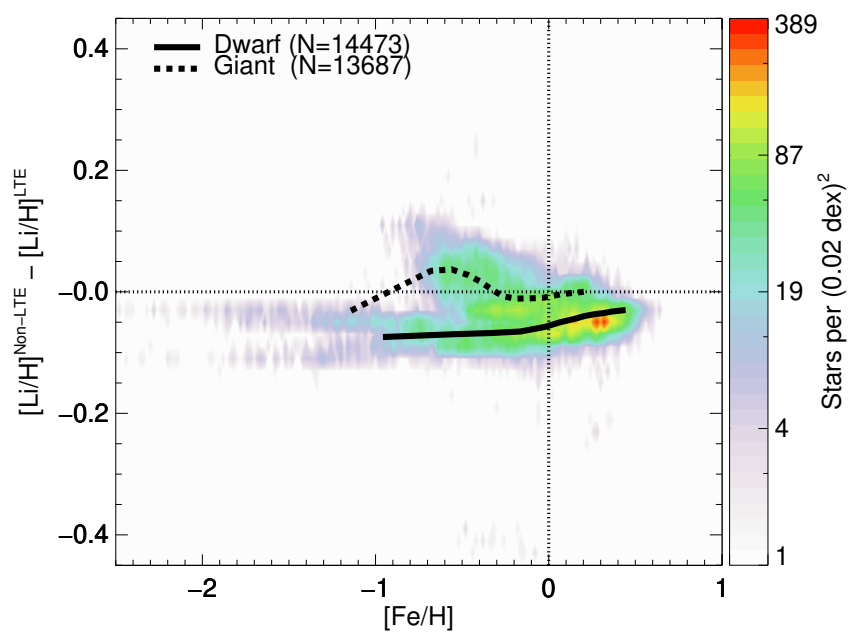

Fig. 3. Differential abundance corrections for lithium. Binned data for dwarfs $\left(\log g / \mathrm{cm} \mathrm{s}^{-2}>3.5\right)$ and giants $\left(\log g / \mathrm{cm} \mathrm{s}^{-2} \leq 3.5\right)$ are overplotted.

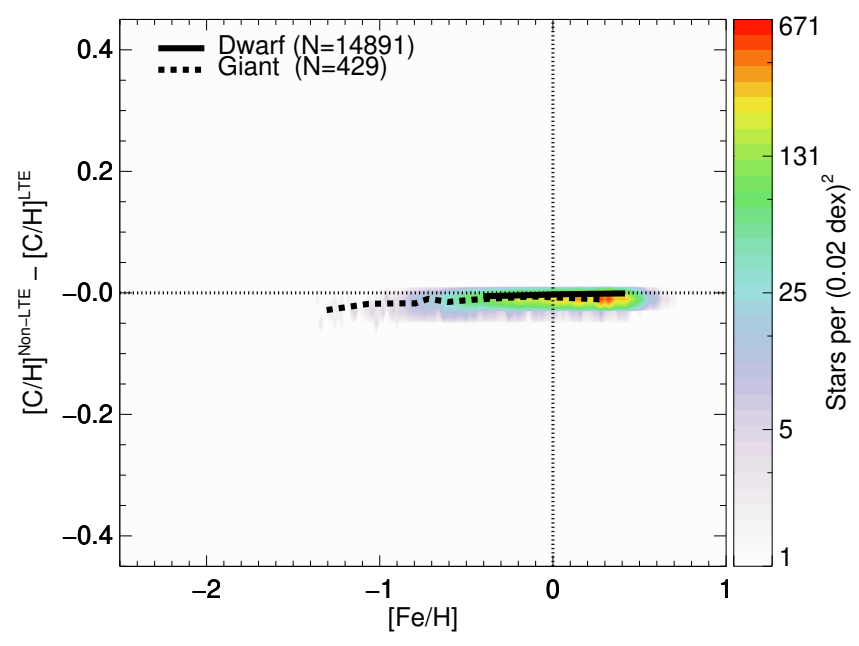

Fig. 4. Differential abundance corrections for carbon. Binned data for dwarfs $\left(\log g / \mathrm{cm} \mathrm{s}^{-2}>3.5\right)$ and giants $\left(\log g / \mathrm{cm} \mathrm{s}^{-2} \leq 3.5\right)$ are overplotted.

Eq. (5)) for the entire sample of up to 50126 stars in Figs. 3-13. We show the abundances themselves in Figs. 14-24: Fig. 14 shows the absolute lithium abundances, $\log \epsilon_{\mathrm{Li}}$, whereas and Figs. 15-24 show the run of $[\mathrm{A} / \mathrm{Fe}]$ from carbon to barium, as functions of the non-LTE iron abundance $[\mathrm{Fe} / \mathrm{H}]$ adopted from GALAH DR3. For a particular element, the plots are restricted to those stars for which both LTE and non-LTE abundances were successfully measured, so the same number of stars are included in both cases. The results were also binned, separately for dwarfs $\left(\log g / \mathrm{cm} \mathrm{s}^{-2}>3.5\right)$ and for giants $\left(\log g / \mathrm{cm} \mathrm{s}^{-2} \leq 3.5\right)$, and these are overplotted to illustrate the average abundance trends. The chemical evolution model of Kobayashi et al. (2020a), discussed in Sect. 5, is overplotted and can be used to compare the LTE and non-LTE trends. For further insight, we also plot various measures of the dispersion in the abundances, in Fig. 25.

There are several features in these figures that help us to gauge the impact of non-LTE effects on large spectroscopic surveys. These include: the severity of the non-LTE abundance corrections (Sect. 4.1); the appearance of the average abundance trends (Sect. 4.2); the differences between the results for dwarfs 


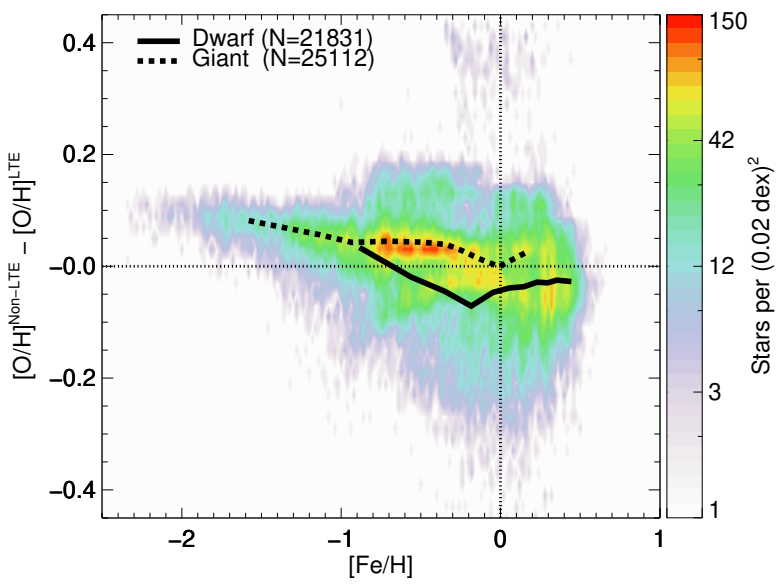

Fig. 5. Differential abundance corrections for oxygen. Binned data for dwarfs $\left(\log g / \mathrm{cm} \mathrm{s}^{-2}>3.5\right)$ and giants $\left(\log g / \mathrm{cm} \mathrm{s}^{-2} \leq 3.5\right)$ are overplotted.

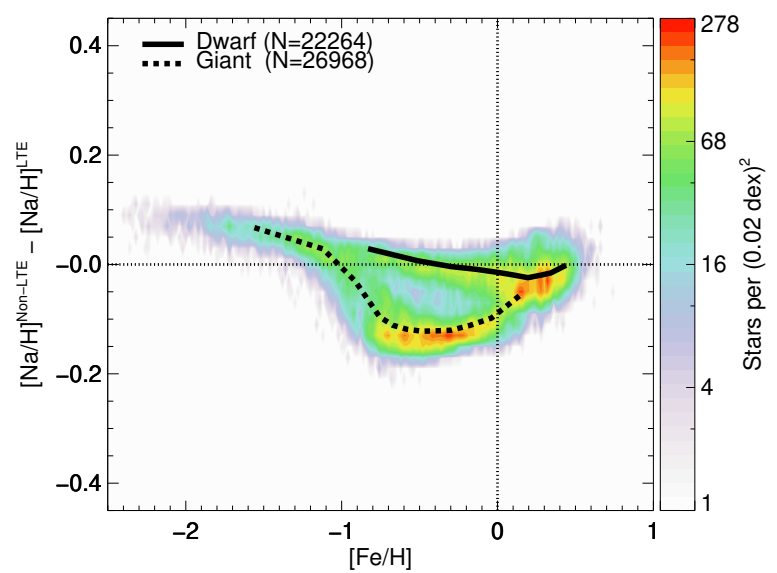

Fig. 6. Differential abundance corrections for sodium. Binned data for dwarfs $\left(\log g / \mathrm{cm} \mathrm{s}^{-2}>3.5\right)$ and giants $\left(\log g / \mathrm{cm} \mathrm{s}^{-2} \leq 3.5\right)$ are overplotted.

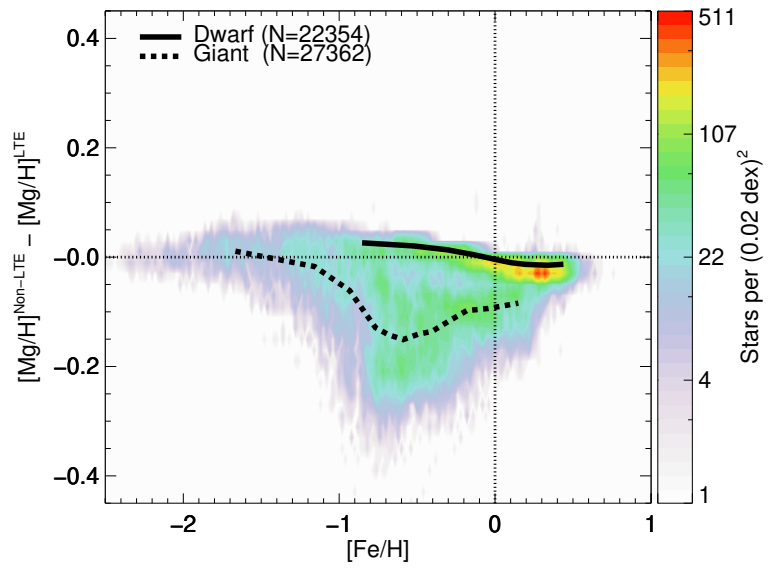

Fig. 7. Differential abundance corrections for magnesium. Binned data for dwarfs $\left(\log g / \mathrm{cm} \mathrm{s}^{-2}>3.5\right)$ and giants $\left(\log g / \mathrm{cm} \mathrm{s}^{-2} \leq 3.5\right)$ are overplotted.

and giants (Sect. 4.3); and the overall dispersions in the elemental abundances (Sect. 4.4). We discuss the implications for the chemical evolution of our Galaxy in Sect. 5.

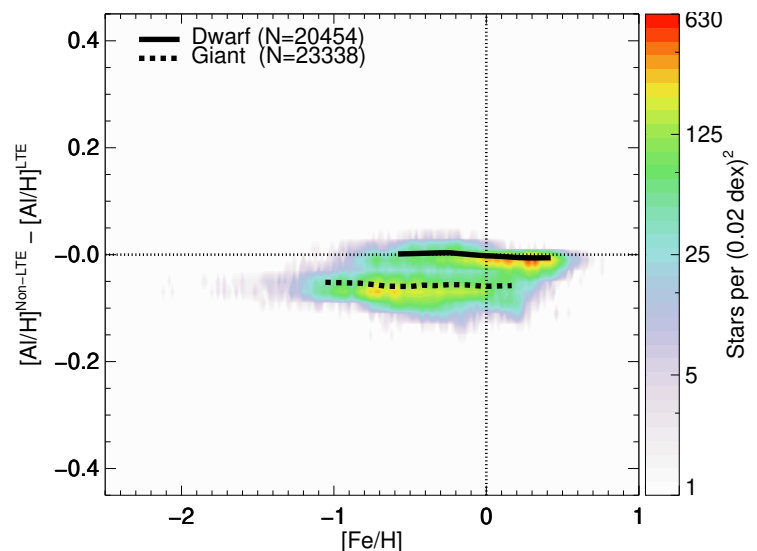

Fig. 8. Differential abundance corrections for aluminium. Binned data for dwarfs $\left(\log g / \mathrm{cm} \mathrm{s}^{-2}>3.5\right)$ and giants $\left(\log g / \mathrm{cm} \mathrm{s}^{-2} \leq 3.5\right)$ are overplotted.

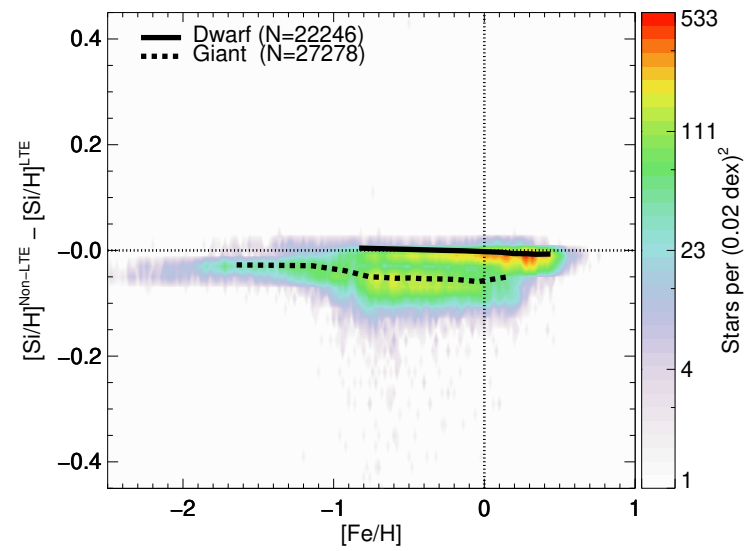

Fig. 9. Differential abundance corrections for silicon. Binned data for dwarfs $\left(\log g / \mathrm{cm} \mathrm{s}^{-2}>3.5\right)$ and giants $\left(\log g / \mathrm{cm} \mathrm{s}^{-2} \leq 3.5\right)$ are overplotted.

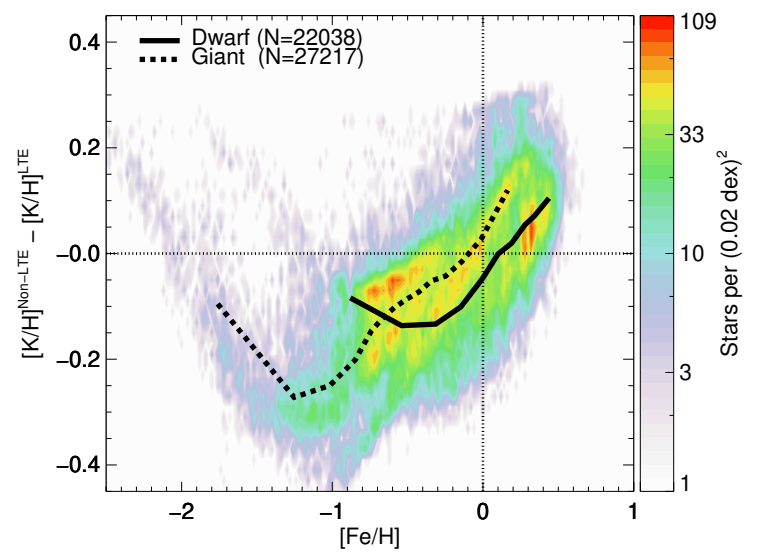

Fig. 10. Differential abundance corrections for potassium. Binned data for dwarfs $\left(\log g / \mathrm{cm} \mathrm{s}^{-2}>3.5\right)$ and giants $\left(\log g / \mathrm{cm} \mathrm{s}^{-2} \leq 3.5\right)$ are overplotted.

\section{Discussion}

\subsection{Typical non-LTE abundance corrections}

In Table 2 we show the typical line-by-line differences between the absolute abundances inferred in non-LTE and in LTE: 


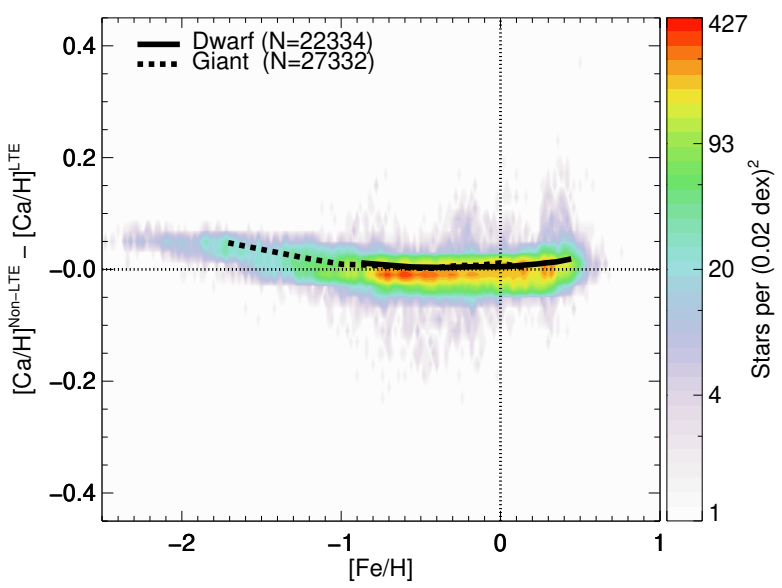

Fig. 11. Differential abundance corrections for calcium. Binned data for dwarfs $\left(\log g / \mathrm{cm} \mathrm{s}^{-2}>3.5\right)$ and giants $\left(\log g / \mathrm{cm} \mathrm{s}^{-2} \leq 3.5\right)$ are overplotted.

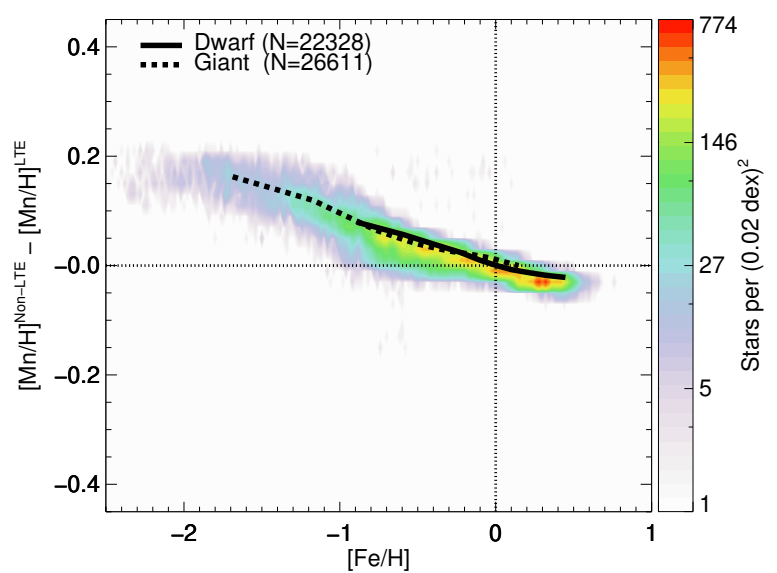

Fig. 12. Differential abundance corrections for manganese. Binned data for dwarfs $\left(\log g / \mathrm{cm} \mathrm{s}^{-2}>3.5\right)$ and giants $\left(\log g / \mathrm{cm} \mathrm{s}^{-2} \leq 3.5\right)$ are overplotted.

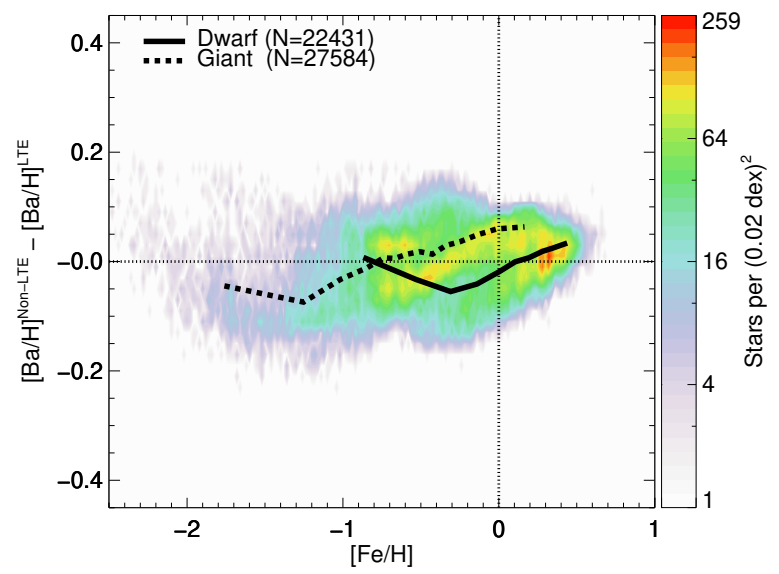

Fig. 13. Differential abundance corrections for barium. Binned data for dwarfs $\left(\log g / \mathrm{cm} \mathrm{s}^{-2}>3.5\right)$ and giants $\left(\log g / \mathrm{cm} \mathrm{s}^{-2} \leq 3.5\right)$ are overplotted.

$\log \epsilon_{\mathrm{A}}{ }^{\text {Non-LTE }}-\log \epsilon_{\mathrm{A}}^{\text {LTE }}$. These are based on the abundances inferred from the present sample, and are shown for different regions of the HR diagram illustrated in Fig. 1 and the abundance plane illustrated in Fig. 2, that is for K dwarfs, F dwarfs, and $\mathrm{K}$ giants, at high and low metallicities.
In metal-rich $\mathrm{F}$ dwarfs, the absolute abundance corrections can be as severe as -0.6 dex for the K I $769.9 \mathrm{~nm}$ resonance line (Reggiani et al. 2019), $-0.5 \mathrm{dex}$ for the O I $777 \mathrm{~nm}$ triplet (Amarsi et al. 2016a), and -0.3 dex for the Ba II $649.7 \mathrm{~nm}$ line (Korotin et al. 2011; Gallagher et al. 2020). Typically the most severe absolute abundance corrections are found for metal-rich $\mathrm{K}$-giants; it is also in this cooler, lower-pressure regime where inelastic hydrogen collisions (Sect. 2.1) play a greater role.

Table 2 shows that for a particular species, the absolute abundance correction goes in the same direction and is of a similar magnitude for the different lines of that species: the non-LTE effects are of a similar nature for the lines of a given species. A consequence of this is that, in the present analysis, non-LTE effects do not average out simply by using multiple lines of the same species. It is beyond the scope of this paper to discuss the non-LTE effects for each of the spectral lines and elements considered here, and instead refer the reader to the papers listed in Table 1 for further details.

It is important to note that with the exception of lithium (Fig. 14), the abundance results presented in Sect. 3.3 are differential in the sense that the LTE and non-LTE solar abundances presented in Table 2 were used to convert absolute elemental abundances $\log \epsilon_{\mathrm{A} l}$ into abundance ratios $[\mathrm{A} / \mathrm{H}]_{l}$ or $[\mathrm{A} / \mathrm{Fe}]_{l}$ relative to the Sun, for given lines $l$. These quantities were then averaged over the different lines $l$, to obtain the final abundance ratios $[\mathrm{A} / \mathrm{H}]$ or $[\mathrm{A} / \mathrm{Fe}]$. The internal normalisation results in a more accurate zero point, and, for the elements with multiple line diagnostics, the line-by-line normalisation leads to lower dispersion, owing to significant cancellation of systematic errors as we discussed in Sect. 3.2.

In Figs. $3-13$ we plot the difference $[\mathrm{A} / \mathrm{H}]^{\text {Non-LTE }}-$ $[\mathrm{A} / \mathrm{H}]^{\mathrm{LTE}}$, or in other words the differences between the nonLTE and LTE panels in Figs. 3-13. This is a rough estimate of the differential abundance corrections (Eq. (5)). The severity of these differential corrections can be less than of the absolute corrections, if the non-LTE effects in the Sun are of the same sign and magnitude as in the star under consideration. For example for oxygen (Fig. 5), the most negative differential corrections reach at most only -0.3 dex compared to the absolute corrections reaching -0.5 dex in metal-rich F-dwarfs, which reflects that the absolute correction in the Sun is -0.2 dex (Table 2). This cancellation is less effective for stars further away from the Sun in parameter space: for example in the cooler giants, as can be seen for both sodium (Fig. 6) and magnesium (Fig. 7); or in the metal-poor regime, as can be seen for manganese (Fig. 12).

\subsection{Non-LTE effects on abundance trends}

We find that the assumption of LTE can lead to average abundance trends that are quantitatively and sometimes even qualitatively different compared to what is found in non-LTE. This is particularly apparent for a few elements: sodium, magnesium, potassium, and manganese, where there are differences in the mean $[\mathrm{A} / \mathrm{Fe}]$ at given $[\mathrm{Fe} / \mathrm{H}]$ of up to $0.2 \mathrm{dex}$, resulting in qualitatively different pictures of the Galactic chemical evolution as we discuss further in Sect. 5.

For sodium (Fig. 17), in LTE, there is a jump in $[\mathrm{Na} / \mathrm{Fe}]$ of from -0.2 dex to +0.2 dex as $[\mathrm{Fe} / \mathrm{H}]$ increases past $-1.0 \mathrm{dex}$. In non-LTE, this jump of $0.4 \mathrm{dex}$ is reduced to just $0.2 \mathrm{dex}$, with $[\mathrm{Na} / \mathrm{Fe}]$ increasing from -0.1 dex to +0.1 dex.

For magnesium (Fig. 18) in LTE there is a clear local maximum in $[\mathrm{Mg} / \mathrm{Fe}]$ of approximately $0.45 \mathrm{dex}$ at $[\mathrm{Fe} / \mathrm{H}] \approx$ -0.75 . In non-LTE, $[\mathrm{Mg} / \mathrm{Fe}]$ shows the usual plateau at low 

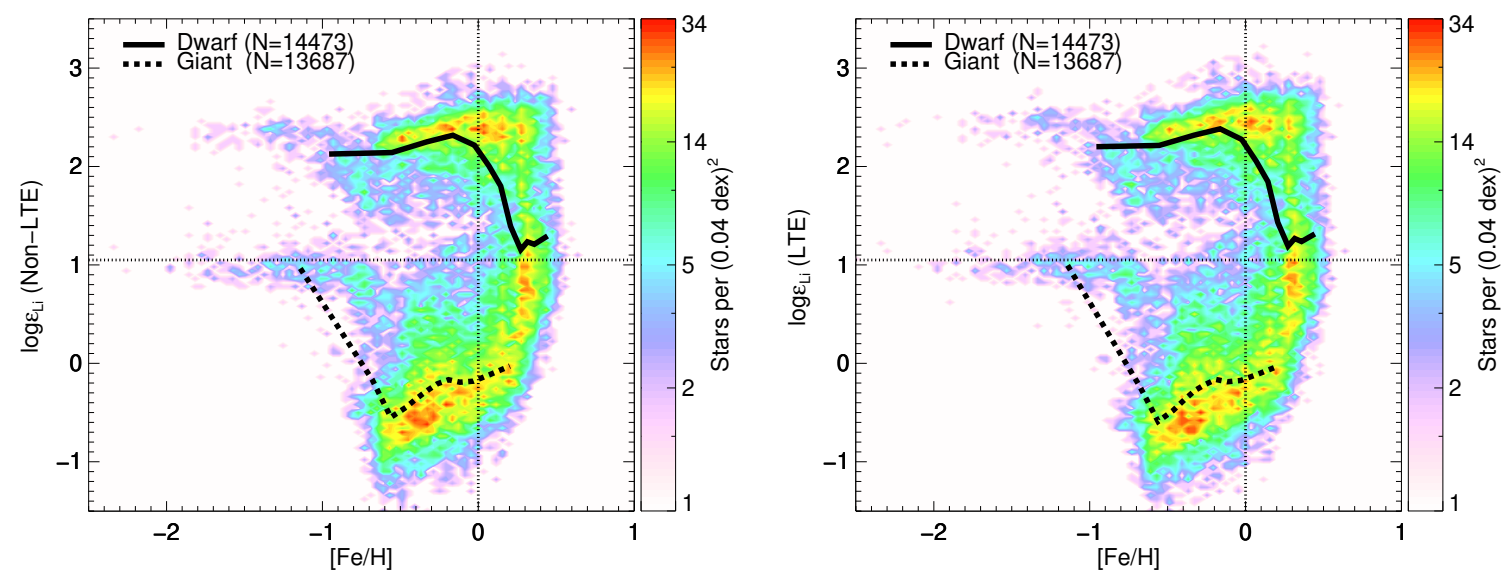

Fig. 14. Non-LTE (left) and LTE (right) lithium abundances, with non-LTE $[\mathrm{Fe} / \mathrm{H}]$ adopted from GALAH DR3 in both cases. Overplotted are binned data for dwarfs $\left(\log g / \mathrm{cm} \mathrm{s}^{-2}>3.5\right)$ and giants $\left(\log g / \mathrm{cm} \mathrm{s}^{-2} \leq 3.5\right)$.
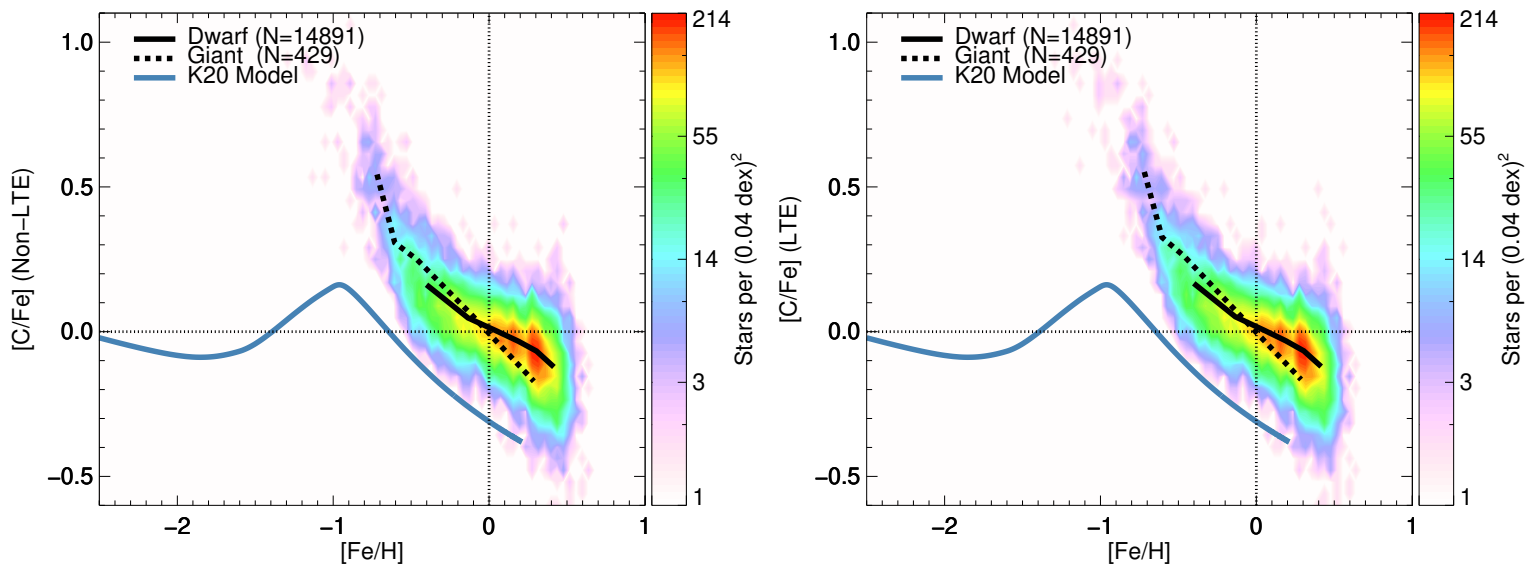

Fig. 15. Non-LTE (left) and LTE (right) carbon abundances, with non-LTE [Fe/H] adopted from GALAH DR3 in both cases. Overplotted are binned data for dwarfs $\left(\log g / \mathrm{cm} \mathrm{s}^{-2}>3.5\right)$ and giants $\left(\log g / \mathrm{cm} \mathrm{s}^{-2} \leq 3.5\right)$, and the GCE model of K20.
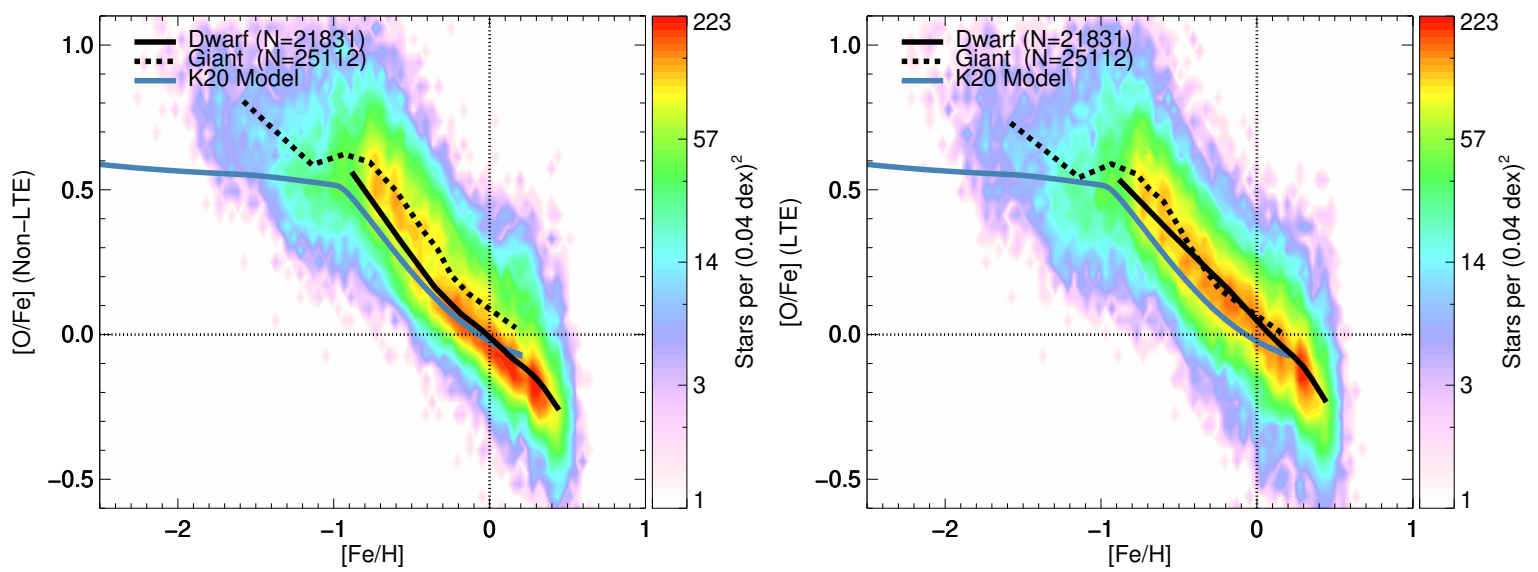

Fig. 16. Non-LTE (left) and LTE (right) oxygen abundances, with non-LTE [Fe/H] adopted from GALAH DR3 in both cases. Overplotted are binned data for dwarfs $\left(\log g / \mathrm{cm} \mathrm{s}^{-2}>3.5\right)$ and giants $\left(\log g / \mathrm{cm} \mathrm{s}^{-2} \leq 3.5\right)$, and the GCE model of K20.

metallicities at $0.25 \mathrm{dex}$, and a decreasing trend towards higher metallicities.

For potassium (Fig. 21), there is also a local maximum in LTE, with $[\mathrm{K} / \mathrm{Fe}]$ peaking at approximately $0.45 \mathrm{dex}$ at $[\mathrm{Fe} / \mathrm{H}] \approx-1.0$. There is also a linear decrease in $[\mathrm{K} / \mathrm{Fe}]$ at super-solar metallicities. In non-LTE, potassium instead shows a trend similar to the $\alpha$-elements, plateauing at $[\mathrm{K} / \mathrm{Fe}] \approx 0.25$ for $[\mathrm{Fe} / \mathrm{H}] \lesssim-1.0$, and showing a flat trend at super-solar metallicities.

Finally for manganese (Fig. 23), in LTE, [Mn/Fe] increases rather steeply from $-0.5 \mathrm{dex}$ at $[\mathrm{Fe} / \mathrm{H}] \approx-2.0$, up to $0.2 \mathrm{dex}$ at $[\mathrm{Fe} / \mathrm{H}] \approx 0.5$. In non-LTE instead, $[\mathrm{Mn} / \mathrm{Fe}]$ plateaus at low 

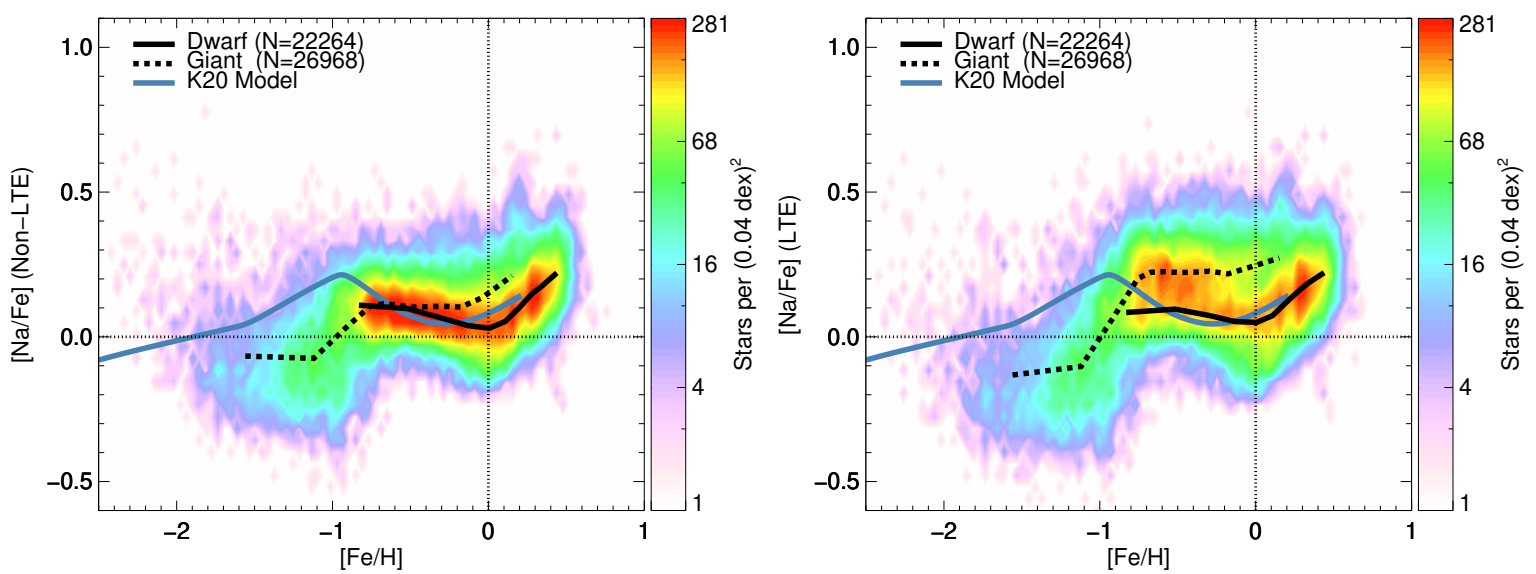

Fig. 17. Non-LTE (left) and LTE (right) sodium abundances, with non-LTE [Fe/H] adopted from GALAH DR3 in both cases. Overplotted are binned data for dwarfs $\left(\log g / \mathrm{cm} \mathrm{s}^{-2}>3.5\right)$ and giants $\left(\log g / \mathrm{cm} \mathrm{s}^{-2} \leq 3.5\right)$, and the GCE model of K20.
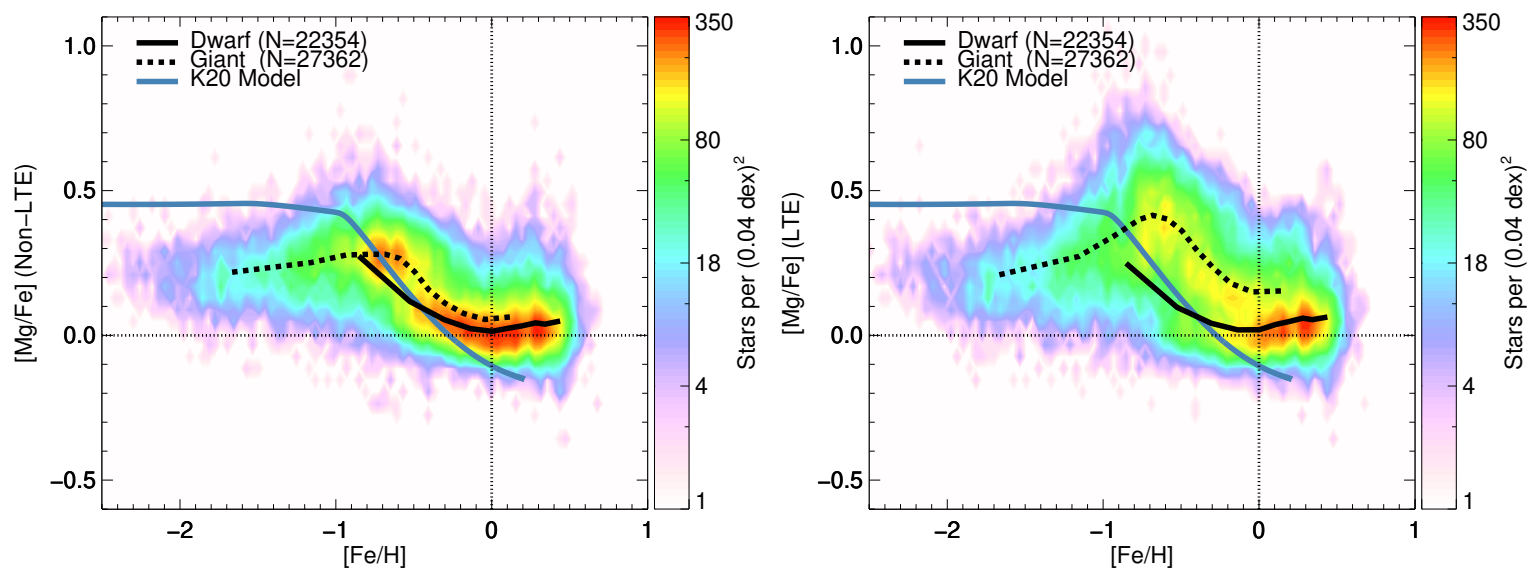

Fig. 18. Non-LTE (left) and LTE (right) magnesium abundances, with non-LTE [Fe/H] adopted from GALAH DR3 in both cases. Overplotted are binned data for dwarfs $\left(\log g / \mathrm{cm} \mathrm{s}^{-2}>3.5\right)$ and giants $\left(\log g / \mathrm{cm} \mathrm{s}^{-2} \leq 3.5\right)$, and the GCE model of K20.
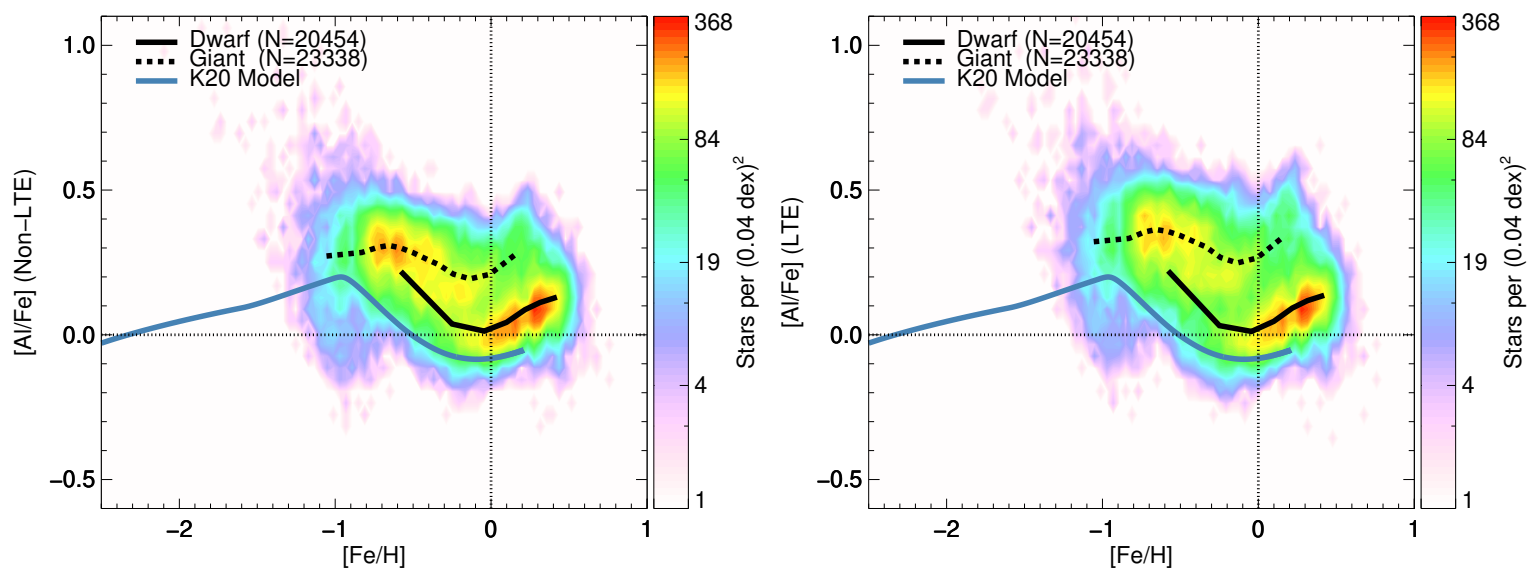

Fig. 19. Non-LTE (left) and LTE (right) aluminium abundances, with non-LTE [Fe/H] adopted from GALAH DR3 in both cases. Overplotted are binned data for dwarfs $\left(\log g / \mathrm{cm} \mathrm{s}^{-2}>3.5\right)$ and giants $\left(\log g / \mathrm{cm} \mathrm{s}^{-2} \leq 3.5\right)$, and the GCE model of K20.

metallicities at approximately -0.3 dex below $[\mathrm{Fe} / \mathrm{H}] \lesssim-1.0$, and the abundance trend is less steep.

\subsection{Non-LTE effects on offsets between dwarfs and giants}

We find that the assumption of LTE can impart large offsets between dwarfs and giants in abundance space (Bonifacio et al.
2009). That is, the giants tend to sit either far above or below the dwarfs in $[\mathrm{A} / \mathrm{Fe}]$ versus $[\mathrm{Fe} / \mathrm{H}]$ space, even if the abundance tracks are parallel to each other, This offset is as large as 0.3 dex in the case of magnesium (Fig. 18); smaller offsets can also be seen for sodium (Fig. 17), silicon (Fig. 20), and potassium (Fig. 21). These offsets shrink significantly for each of these elements when non-LTE methods are used. For example, for sodium 

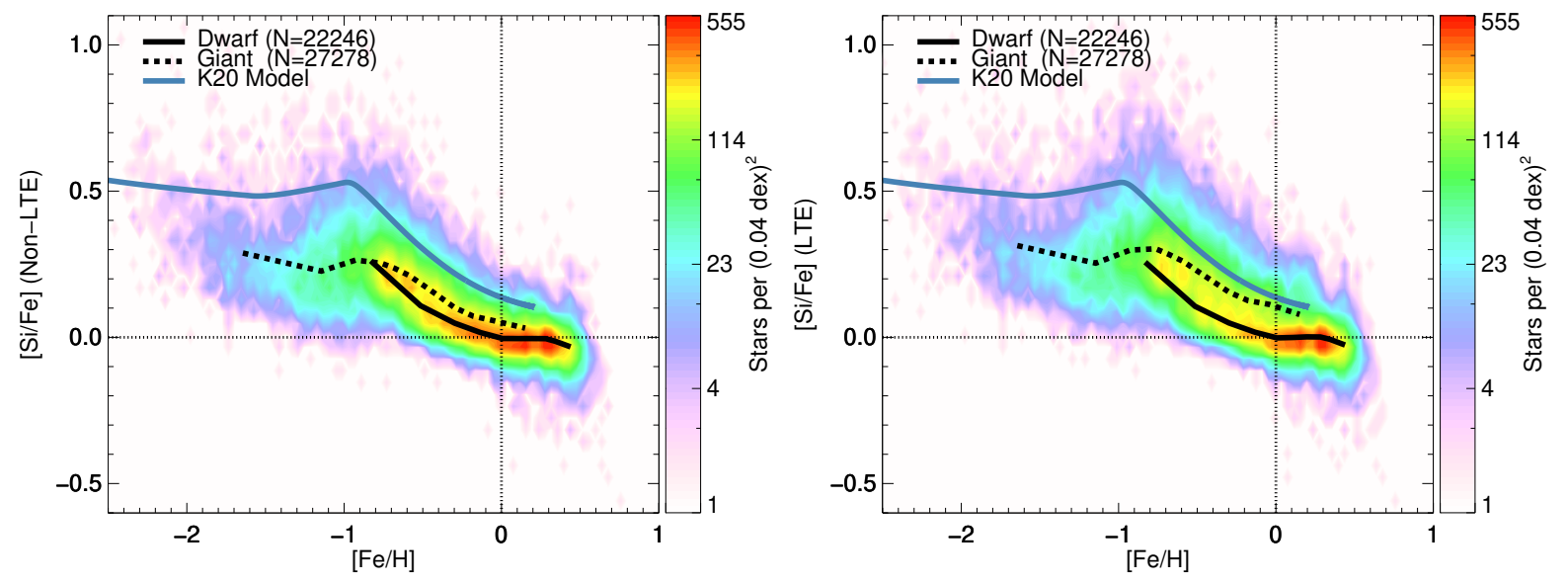

Fig. 20. Non-LTE (left) and LTE (right) silicon abundances, with non-LTE [Fe/H] adopted from GALAH DR3 in both cases. Overplotted are binned data for dwarfs $\left(\log g / \mathrm{cm} \mathrm{s}^{-2}>3.5\right)$ and giants $\left(\log g / \mathrm{cm} \mathrm{s}^{-2} \leq 3.5\right)$, and the GCE model of K20.
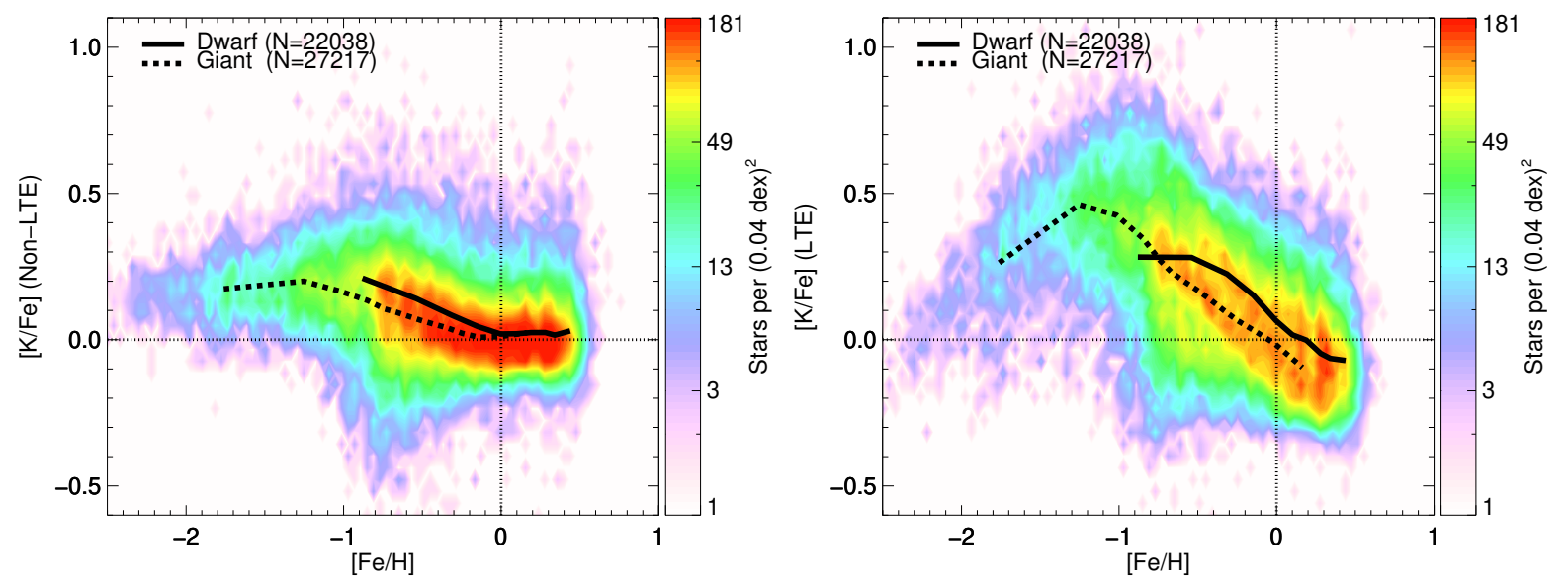

Fig. 21. Non-LTE (left) and LTE (right) potassium abundances, with non-LTE [Fe/H] adopted from GALAH DR3 in both cases. Overplotted are binned data for dwarfs $\left(\log g / \mathrm{cm} \mathrm{s}^{-2}>3.5\right)$ and giants $\left(\log g / \mathrm{cm} \mathrm{s}^{-2} \leq 3.5\right)$. The GCE model of K20 falls below the vertical axis of this plot.
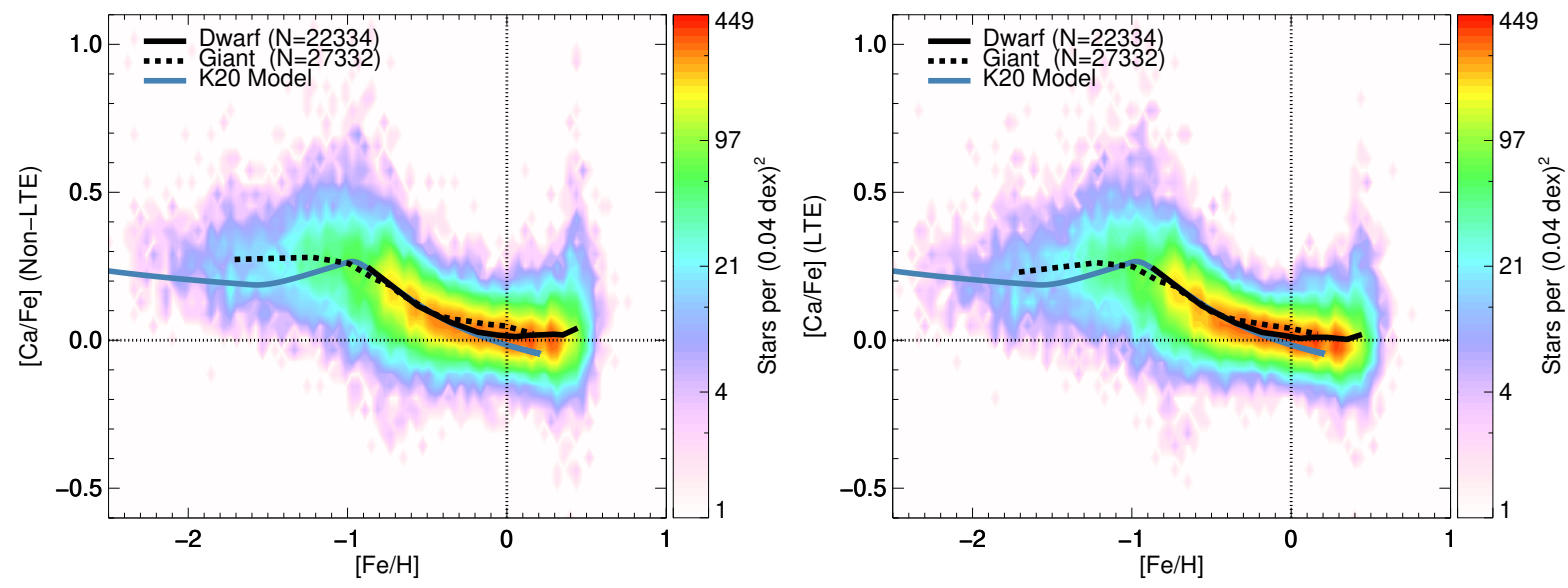

Fig. 22. Non-LTE (left) and LTE (right) calcium abundances, with non-LTE [Fe/H] adopted from GALAH DR3 in both cases. Overplotted are binned data for dwarfs $\left(\log g / \mathrm{cm} \mathrm{s}^{-2}>3.5\right)$ and giants $\left(\log g / \mathrm{cm} \mathrm{s}^{-2} \leq 3.5\right)$, and the GCE model of K20.

it is reduced by 0.2 dex, and the tracks are in good agreement between $-1 \lesssim[\mathrm{Fe} / \mathrm{H}] \lesssim-0.5$.

For some elements, taking non-LTE effects into account does not negate entirely the offsets between the dwarfs and giants. The residual offsets for most elements tend to be only of the order 0.05 dex. For aluminium (Fig. 19) and barium (Fig. 24), the offsets reach 0.2 dex. These large offsets may reflect other systematic errors within the GALAH analysis pipeline, even though the different $\mathrm{Al} \mathrm{I}$ and $\mathrm{Ba}$ II lines are in reasonable agreement (the line-by-line dispersion is not anomalously large for aluminium and barium in Fig. 24). For extremely metal-poor stars Andrievsky et al. (2008) and Bonifacio et al. (2009) found 
A. M. Amarsi et al.: Non-LTE departure coefficients for large spectroscopic surveys
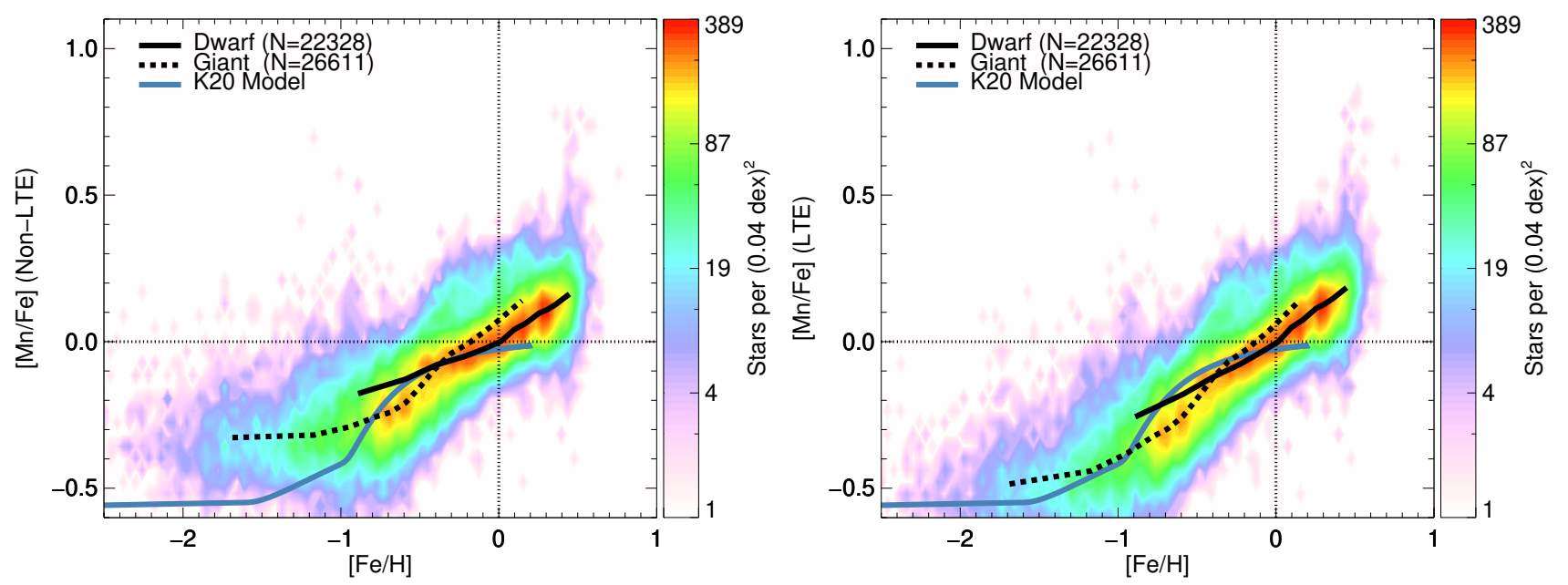

Fig. 23. Non-LTE (left) and LTE (right) manganese abundances, with non-LTE [Fe/H] adopted from GALAH DR3 in both cases. Overplotted are binned data for dwarfs $\left(\log g / \mathrm{cm} \mathrm{s}^{-2}>3.5\right)$ and giants $\left(\log g / \mathrm{cm} \mathrm{s}^{-2} \leq 3.5\right)$, and the GCE model of K20.
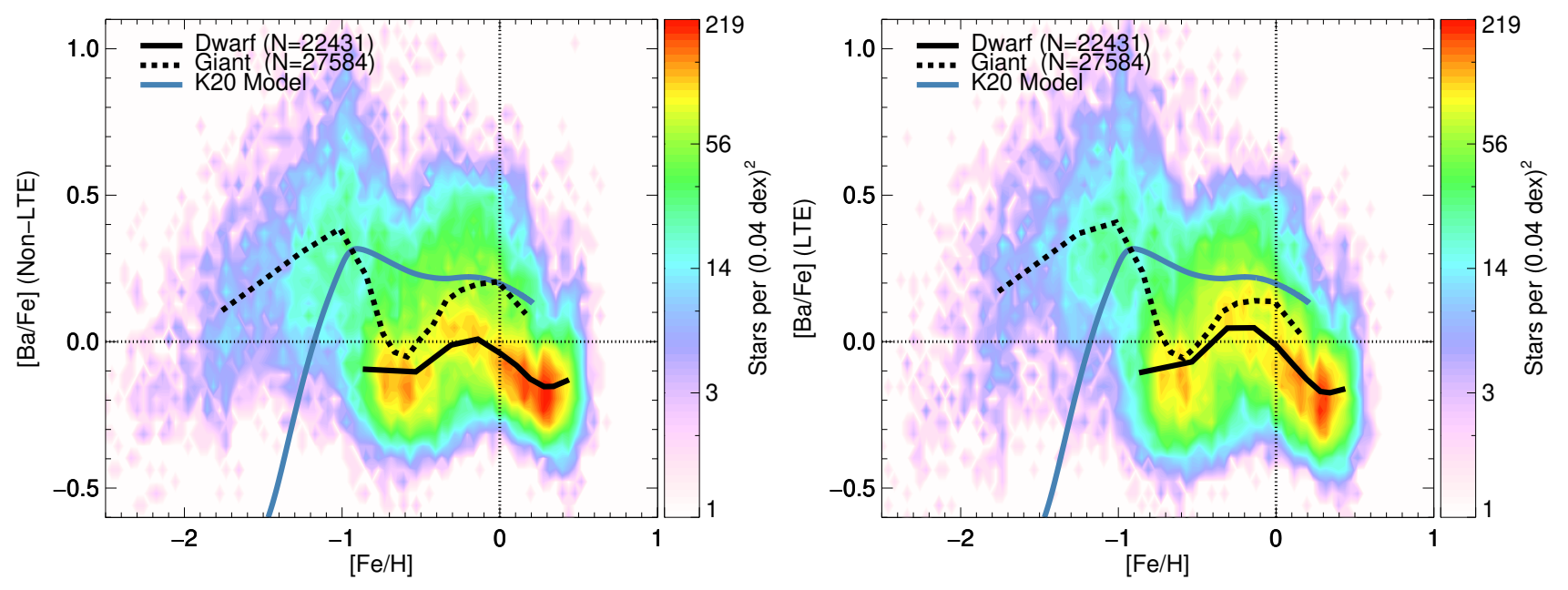

Fig. 24. Non-LTE (left) and LTE (right) barium abundances, with non-LTE [Fe/H] adopted from GALAH DR3 in both cases. Overplotted are binned data for dwarfs $\left(\log g / \mathrm{cm} \mathrm{s}^{-2}>3.5\right)$ and giants $\left(\log g / \mathrm{cm} \mathrm{s}^{-2} \leq 3.5\right)$, and the GCE model of K20.
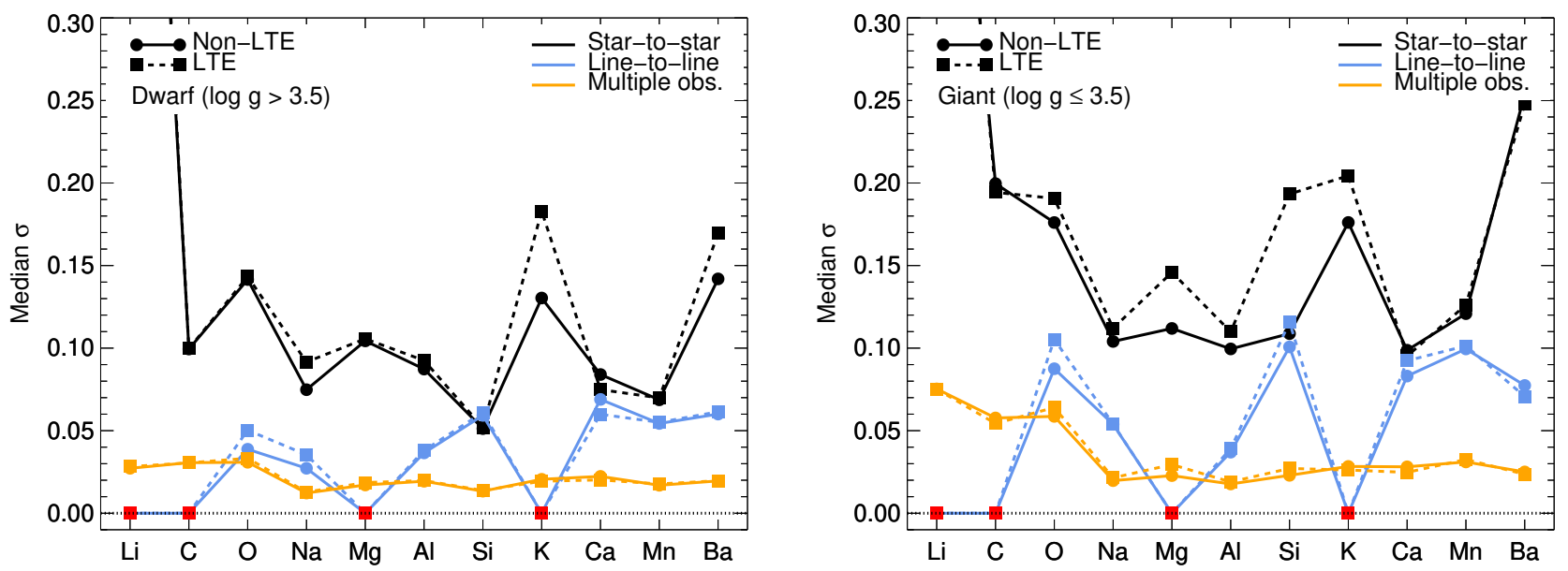

Fig. 25. Dispersions in the abundance results, in non-LTE (solid) and LTE (dashed) for dwarfs $\left(l e f t ; \log g / \mathrm{cm} \mathrm{s}^{-2}>3.5\right)$ and giants (right; $\left.\log g / \mathrm{cm} \mathrm{s}^{-2} \leq 3.5\right)$. "Multiple obs." (orange) show median standard deviations in $[\mathrm{A} / \mathrm{Fe}]$ over multiple observations of the same stars. "Lineto-line" (blue) show the median standard deviations in $[\mathrm{A} / \mathrm{Fe}]$ over multiple spectral lines for the same star; lithium, carbon, magnesium, and potassium in red are set to zero because they were inferred from single lines. "Star-to-star" (black) show the median standard deviation in [A/Fe] (or in $\log \epsilon_{\mathrm{A}}$, in the case of lithium) across the different bins shown in Figs. 14-24. 
that taking non-LTE effects into account bring the aluminium abundances of dwarfs and giants into agreement, which might indicate that the offsets seen here at higher metallicities are not physical.

For the $\alpha$-elements oxygen (Fig. 16), magnesium (Fig. 18), and silicon (Fig. 20), the residual offsets in non-LTE amount to approximately 0.1 dex. These offsets may in part be due to selection effects: GALAH is a magnitude limited survey, and the giants are brighter and observed to larger distances, and thus probe more of the Galactic thick disc, that is typically $\alpha$-rich. Conversely, the dwarfs probe more of the Galactic thin disc, that is typically $\alpha$-poor (Hayden et al. 2015; Feuillet et al. 2019). However, one would then expect a similar offset for calcium (Fig. 22), whereas instead the mean trends for the dwarfs and giants lie on top of each other. This may reflect residual systematics with the non-LTE calcium abundances. For instance, Osorio et al. (2020) showed recently that non-LTE Mg I continuous opacities can make a significant impact on the predicted $\mathrm{Ca}$ I departure coefficients; this effect is not taken into account in the present calculations.

\subsection{Non-LTE effects on abundance dispersions}

We find that the assumption of LTE can substantially increase the spread of $[\mathrm{A} / \mathrm{Fe}]$ at given $[\mathrm{Fe} / \mathrm{H}]$. This is immediately evident by comparing the densities of stars in LTE and non-LTE in many of the plots presented in in Sect. 3.3, and is particularly obvious for oxygen (Fig. 16), sodium (Fig. 17), magnesium (Fig. 18), and potassium (Fig. 21).

The dispersions in the stellar elemental abundances can also be quantified. In Fig. 25 we show the median standard deviation in $[\mathrm{A} / \mathrm{Fe}]$ (or for lithium, $\log \epsilon_{\mathrm{Li}}$ ) across the different bins shown in Figs. 14-24, separately for dwarfs, and for giants; this is labelled "Star-to-star". For comparison, also plotted are the median standard deviations in $[\mathrm{A} / \mathrm{Fe}]$ over multiple spectral lines, as inferred for a given element and for a given star (after already averaging over multiple observations, if necessary); this is labelled "Line-to-line". Another comparison can be made, by noting that there are 3237 stars in the sample of 50126 for which two or more observations exist. Thus, the median standard deviations in $[\mathrm{A} / \mathrm{Fe}]$ over multiple observations of the same stars are also plotted; this is labelled "Multiple obs.". The stipulated signal-to-noise ratios of this sample is in close agreement to that of the entire sample.

The assumption of LTE does not significantly impact the dispersions in the results from multiple observations. This reflects the random noise that are propagated from the finite signal-tonoise ratios of the observations into the inferred abundances, either directly or via the inferred stellar parameters. In Fig. 25 the dispersions are approximately 0.02 dex in dwarfs and 0.03 dex in giants for most elements, in both LTE and non-LTE; they are slightly larger for lithium, carbon, and oxygen, namely $0.03 \mathrm{dex}$ in dwarfs, and 0.06 dex in giants. These small dispersions reflect the great potential of GALAH data for high-precision studies of stellar abundances and Galactic chemical evolution.

The assumption of LTE has a small impact on the lineto-line dispersions. This dispersion reflects (in addition to the random noise) the systematic errors arising from the pipeline, arising from deficiencies in modelling the stellar spectra including 3D and non-LTE effects, as well as the impact of blends, and consistent offsets in the continuum placement. We caution that the entirety of the systematic errors are not represented here, because the spectral lines being used for a particular element belong to the same ionisation stage and also tend to have similar wavelengths and excitation potentials (Table 2), and thus tend to have similar sensitivities to systematic errors in the stellar parameters, and suffer similar 3D and non-LTE effects. In Fig. 25 the dispersions are, in non-LTE, approximately 0.05 dex for dwarfs, and 0.07 dex for giants. In LTE, they are typically only about 0.01 dex larger, owing to the cancellation effects obtained by using line-by-line solar abundances (Sect. 3.2).

Finally, the assumption of LTE does significantly impact the star-to-star dispersions. This reflects random noise and systematic errors in the pipeline, as well as intrinsic dispersion arising from different stars with the same $[\mathrm{Fe} / \mathrm{H}]$ actually having different values of $[\mathrm{A} / \mathrm{Fe}]$ in their atmospheres. The systematic errors are better represented here than in the line-to-line dispersions. In Fig. 25, in non-LTE and for most elements, the dispersions are approximately 0.05 to 0.15 dex for dwarfs and 0.1 to 0.2 dex for giants, much larger than the dispersions in the results from multiple observations and also typically larger than the line-toline dispersions. In LTE the star-to-star dispersions can be much larger: the largest difference is for magnesium and silicon in giants, where the dispersion is increased by 0.1 dex.

It is difficult to make general conclusions about whether or not the non-LTE results shown in Figs. 14-24 are dominated by intrinsic dispersion, or are dominated by systematics errors. The small dispersions in the results from multiple observations in Fig. 25 suggests that random noise are not dominating the dispersions in the abundance relations. For lithium (Fig. 14), intrinsic dispersion is clearly dominant; lithium is fragile and easily destroyed in stellar atmospheres. Some elements such as silicon (Fig. 20) show tight abundance relations in non-LTE, with star-to-star dispersions that are similar to the line-to-line dispersions in Fig. 25, which suggests that systematics could be the limiting factor here. For other elements with broader spreads in the abundance relations including aluminium (Fig. 19) and barium (Fig. 24), intrinsic effects could be dominant, although residual systematic errors still cannot be ruled out as discussed above.

\section{Galactic chemical evolution and comparison with previous studies}

In this section we briefly discuss the non-LTE abundance trends (Figs. 14-24), and contrast them with results recently presented elsewhere in the literature. For elements heavier than lithium, we compare the observed trends to the standard Galactic chemical evolution model of the solar neighbourhood presented in Kobayashi et al. (2020a), hereafter referred to as the K20 model. In brief, the K20 model assumes instantaneous mixing in the interstellar medium (a one-zone model), adopting an initial mass function from Kroupa (2008). Enrichment by intermediatemass and massive stars via winds and core collapse supernova, neutron-star mergers, and Type Ia supernova are considered: for Type Ia supernovae, the progenitor model and nucleosynthesis yields from Kobayashi et al. (2020b) are adopted. The K20 model is based on theoretical nucleosynthesis yields and event rates and avoids empirical relations and calibrations; in particular, the zero-points of the model have not been adjusted.

\subsection{Lithium}

Lithium was one of th few nuclei that formed during the Big Bang (Fields 2011), and so lithium abundances impose constraints on the primordial nucleosynthesis and baryon density of the early Universe. Later in cosmic time lithium may be produced by spallation reactions in cosmic rays and by various 
stellar sources (Prantzos 2012). As a fragile element, lithium can be destroyed by proton capture reactions at relatively low temperatures in the stellar interior, making it a sensitive tracer of mixing within stars (Pinsonneault 1997). However, recent observations have shown that surface lithium abundances could also be enriched, by multiple mechanisms that act in different parts of the HR diagram (Gao et al. 2020; Martell et al. 2020).

The lithium abundances here confirm those recently presented in the literature. Figure 14 shows a large intrinsic spread of abundances, with the dwarfs and giants following two distinct trends: in giant stars the convection zones extend deeper into the stellar interior, to high enough temperatures for lithium to be burnt. The most metal-poor dwarfs in the sample converge to the well-known "Spite-plateau" (Spite \& Spite 1982). In nonLTE, this is at $\log \epsilon_{\mathrm{Li}} \approx 2.1$. In LTE this is about 0.1 dex higher, as the abundance corrections for the Li I $670.8 \mathrm{~nm}$ line is slightly negative and not too sensitive to metallicity (Fig. 3). In both LTE and non-LTE, there is a mild increase in $\log \epsilon_{\mathrm{Li}}$ with increasing $[\mathrm{Fe} / \mathrm{H}]$ indicative of a gradual enrichment of lithium in the Galaxy as found in earlier studies of dwarf stars (Bensby \& Lind 2018).

Recent studies have reported a puzzling drop in $\log \epsilon_{\mathrm{Li}}$ at super-solar metallicities (Delgado Mena et al. 2015; Stonkute et al. 2020). The same trend is found in the present study, in both LTE and non-LTE. It has been proposed that this is a signature of radial migration (Guiglion et al. 2019). According to this picture, towards higher metallicities the sample is dominated by stars that were born closer to the Galactic centre, in a more metal-rich environment. To have reached the solar neighbourhood, these stars have to be old, implying that they have had time to deplete significant amounts of their birth lithium abundance.

\subsection{Carbon and oxygen}

Carbon is a light element that traces multiple sources including Asymptotic Giant Branch (AGB) stars, core-collapse supernova, and Wolf-Rayet stars (Karakas \& Lattanzio 2014; Romano et al. 2019, 2020). Some molecular features are present in the GALAH spectral range and can be used to infer carbon abundances in cooler stars to low metallicities (Čotar et al. 2019). However in the present study, carbon abundances were determined from the high-excitation C I $658.8 \mathrm{~nm}$ line that is usually too weak to be observed in cool giant stars. Thus the steep trend seen in Fig. 15 at low metallicity is based on a relatively small number of giant stars, and may be due to the GALAH analysis pipeline interpreting noise as a detected line; it should be treated with scepticism. The Galactic evolution of carbon inferred here is based on the warmer dwarf stars: Fig. 15 shows a mild linear decrease in $[\mathrm{C} / \mathrm{Fe}]$ from approximately $0.2 \mathrm{dex}$ at $[\mathrm{Fe} / \mathrm{H}] \approx$ -0.5 , to -0.1 dex at $[\mathrm{Fe} / \mathrm{H}] \approx+0.5$. This likely reflects that the cosmic production of iron from Type Ia supernova outpaces that of carbon from AGB stars or from massive stars. The trend is very similar in LTE owing to very mild abundance corrections for the C I $658.8 \mathrm{~nm}$ line (Fig. 4).

Oxygen is an $\alpha$-element that forms almost entirely via hydrostatic burning in massive stars (Kobayashi et al. 2006). The dwarfs and giants form parallel tracks in $[\mathrm{O} / \mathrm{Fe}]$ versus $[\mathrm{Fe} / \mathrm{H}]$ in Fig. 16, albeit offset by approximately 0.05 to 0.10 dex. This offset may reflect selection effects: the giants probe more of the $\alpha$-rich Galactic thick disc (Sect. 4.3). There is a steep linear decrease in $[\mathrm{O} / \mathrm{Fe}]$ from $0.5 \mathrm{dex}$ at $[\mathrm{Fe} / \mathrm{H}] \approx-1.0$, down to below -0.2 dex at $[\mathrm{Fe} / \mathrm{H}] \approx 0.5$, that reflects that the cosmic production of iron from Type Ia supernova vastly outweighs that of oxygen from massive stars at high metallicities. In LTE, the abundance gradient is similar, however the dwarf trends are offset to higher abundances; the LTE results imply that the Sun is underabundant in oxygen.

In general at moderate metallicities these results for carbon and oxygen are consistent with those from previous highresolution, high signal-to-noise ratio studies in LTE and in non-LTE (Bensby et al. 2014; Zhao et al. 2016; Amarsi et al. 2019b; Stonkute et al. 2020). The gradients in the abundance trends are also captured well in the K20 model; for oxygen the non-LTE results are clearly in better agreement than the LTE results. However they are not quite consistent with results for giants from APOGEE; these tend to find much flatter relationships, with a plateau in $[\mathrm{O} / \mathrm{Fe}]$ or even a slight increase in $[\mathrm{C} / \mathrm{Fe}]$ at super-solar metallicities (Hayes et al. 2018). The APOGEE abundances are inferred in LTE from molecular lines, prone to different systematics (in particular from 3D effects; Collet et al. 2007), and this may be the reason for the discrepancies.

In the more metal-poor regime, $[\mathrm{O} / \mathrm{Fe}]$ still shows a linear decrease with increasing $[\mathrm{Fe} / \mathrm{H}]$, albeit with a more gentle slope, as well as a hint of a plateau at $[\mathrm{Fe} / \mathrm{H}] \approx-1.0$, The very high values of $[\mathrm{O} / \mathrm{Fe}]$ at the lowest metallicities is difficult to reconcile with the canonical picture of a $\alpha$-abundance plateau at low metallicities, as also seen in the $\mathrm{K} 20$ model below $[\mathrm{Fe} / \mathrm{H}] \lesssim$ -1.0 . However it is qualitatively consistent with what was found in earlier non-LTE studies of the O I $777 \mathrm{~nm}$ triplet (Ramírez et al. 2012; Amarsi et al. 2019b). Similar to carbon, the metalpoor trend is driven by the giants and should also be treated with scepticism because the high-excitation triplet is very weak in this regime. One or two of the weaker components becoming immeasurable over the noise, and perhaps spuriously measured, may also explain the relatively large star-to-star and line-to-line dispersions seen for oxygen in the giant stars in Fig. 25.

\subsection{Sodium, aluminium, and potassium}

Sodium, aluminium, and potassium are light elements with odd proton numbers, formed primarily in hydrostatic burning in massive stars with metallicity-dependent yields (Kobayashi et al. 2006). In Fig. 17 the non-LTE analysis indicates that $[\mathrm{Na} / \mathrm{Fe}]$ plateaus at approximately $-0.1 \mathrm{dex}$ at $[\mathrm{Fe} / \mathrm{H}] \lesssim-1.0$, and then jumps by 0.2 dex. Between $-1.0 \lesssim[\mathrm{Fe} / \mathrm{H}] \lesssim 0.0$, sodium abundances show a gentle decrease from 0.1 dex down to 0.0 dex, that can be attributed to the onset of Type Ia supernova. At supersolar metallicities, $[\mathrm{Na} / \mathrm{Fe}]$ increases again, reaching $0.25 \mathrm{dex}$ at $[\mathrm{Fe} / \mathrm{H}] \approx 0.5$. As discuss in Sect. 4.2, the trend from the LTE analysis is qualitatively similar, however with a much larger jump at low metallicity.

At sub-solar metallicities, aluminium and potassium behave similarly to the $\alpha$-elements (Sect. 5.4). In Fig. 19, in both LTE and non-LTE, there appears to be an aluminium-poor component with $[\mathrm{Al} / \mathrm{Fe}] \approx 0.0$ at $[\mathrm{Fe} / \mathrm{H}] \approx 0.0$, and an aluminium-rich component with $[\mathrm{Al} / \mathrm{Fe}] \approx 0.25$ at $[\mathrm{Fe} / \mathrm{H}] \approx-1.0$, (traced by dwarfs and giants respectively; Sect. 4.3). Similarly, in Fig. 21 [K/Fe] shows a plateau at approximately 0.25 dex below $[\mathrm{Fe} / \mathrm{H}] \lesssim-1.0$, and a mild decrease down to $[\mathrm{Fe} / \mathrm{H}] \approx 0.0$. This plateau is significantly higher, and the trend much steeper, in the LTE plot (Sect. 4.2). At super-solar metallicities aluminium behaves qualitatively similar to sodium: in the dwarfs, $[\mathrm{Al} / \mathrm{Fe}]$ increases from $0.0 \mathrm{dex}$ at $[\mathrm{Fe} / \mathrm{H}] \approx 0.0$, to $0.2 \mathrm{dex}$ at $[\mathrm{Fe} / \mathrm{H}] \approx 0.5$. In contrast, at these metallicities potassium shows a flat trend, similar to the canonical $\alpha$-elements magnesium, silicon, and calcium (Sect. 5.4).

The observed run of $[\mathrm{Na} / \mathrm{Fe}]$ is in qualitative agreement with what has been found in other recent high-resolution, high 
signal-to-noise ratio non-LTE studies (Bensby et al. 2014; Zhao et al. 2016). The aluminium and potassium abundances agree well with LTE results for giants from APOGEE (Hayes et al. $2018)$, but the increasing trend of $[\mathrm{Al} / \mathrm{Fe}]$ at super-solar metallicities is not seen in LTE in Bensby et al. (2014) nor in non-LTE in Zhao et al. (2016). The K20 model quantitatively reproduces the sodium abundances, and the shape of the $[\mathrm{Al} / \mathrm{Fe}]$ trend for dwarfs. However, as with most GCE models (Reggiani et al. 2019) the K20 model significantly underestimates the cosmic abundance of potassium.

As pointed out already in Sect. 4.3, there is an offset between the dwarfs and giants for aluminium of 0.2 dex. Since the dwarf and giant trends have similar shapes, any such offset could be calibrated away in APOGEE results, which are strictly for giants. A similar offset between dwarfs and giants was found in LTE in the Gaia-ESO survey (Smiljanic et al. 2016); the authors suspected the offset was due to systematics errors, albeit not related to departures from LTE. In the present study, we similarly suspect that the offset signals residual systematic errors in the aluminium abundances, and thus that our results should be treated with caution.

\subsection{Magnesium, silicon, and calcium}

Like oxygen, these canonical $\alpha$-elements form primarily through hydrostatic burning in massive stars, however the yields are altered during explosive nucleosynthesis (Kobayashi et al. 2006). The non-LTE results for magnesium, silicon, and calcium each display a plateau at low metallicities, and a knee at $[\mathrm{Fe} / \mathrm{H}] \approx$ -1.0 that signals the onset of Type Ia supernova (McWilliam 1997). Here this is visible via the dichotomy between the $\alpha$-poor thin disc and the $\alpha$-rich thick disc (traced by dwarfs and giants respectively; Sect. 4.3). In Figs. 18, 20, and 22, this thick disc forms a plateau at approximately $[\alpha / \mathrm{Fe}] \approx 0.25$ below $[\mathrm{Fe} / \mathrm{H}] \lesssim$ -1.0 . At super-solar metallicities these elements tend to show flat trends, with abundance ratios that are close to solar. For silicon and calcium, the LTE trends are qualitatively similar to the nonLTE ones, but for magnesium the LTE abundances show a local maximum rather than a plateau, as discussed in Sect. 4.2, that would be difficult to reconcile with our standard understanding of Galactic chemical evolution.

The non-LTE results are quantitatively consistent with recent high-resolution, high signal-to-noise ratio non-LTE studies (Zhao et al. 2016; Bergemann et al. 2017a,b; Mashonkina et al. 2019). Is interesting to also compare them with LTE results inferred from from giants in APOGEE (Hayes et al. 2018), as that study is based on a large sample size. Below $[\mathrm{Fe} / \mathrm{H}]<-0.9$ the authors separate their stars into those having low $(\mathrm{LMg})$ and high $(\mathrm{HMg})$ magnesium abundances. The LMg population, possibly accreted from dwarf galaxies (Nissen \& Schuster 2010), shows a linear decrease in $[\alpha / \mathrm{Fe}]$ with $[\mathrm{Fe} / \mathrm{H}]$, whilst the $\mathrm{HMg}$ population, probably inherent to our Galaxy, shows a plateau at approximately 0.3 dex. These two populations may well be hidden under the systematics of our analysis (Sect. 4.4), and we note that the dispersion in Figs. 18, 20, and 22 do visibly increase below $[\mathrm{Fe} / \mathrm{H}] \lesssim-1.0$.

The K20 model is in qualitative agreement with the plateaus seen in the non-LTE results. Quantitatively, however, there are some important discrepancies. The model overestimates the amount of magnesium and silicon in the metal-poor Galaxy, with plateaus of $[\mathrm{Mg} / \mathrm{Fe}] \approx 0.5$ and $[\mathrm{Si} / \mathrm{Fe}] \approx 0.5$, about $0.25 \mathrm{dex}$ larger than what is observed. In contrast, the K20 model shows good agreement for calcium, with a plateau of $[\mathrm{Ca} / \mathrm{Fe}] \approx 0.25$. Compared with the other elements, it is much harder to change the relative ratios among $\alpha$-elements. It might be reflect the failure of 1D hydrostatic supernova models (Müller 2016), or perhaps uncertainties in the nuclear reaction rates (deBoer et al. 2017; Fields et al. 2018).

\subsection{Manganese}

Manganese is an iron-peak element that is important for constraining the physics of Type Ia supernova. More manganese than iron is produced in these events, resulting in a positive trend of $[\mathrm{Mn} / \mathrm{Fe}]$ with $[\mathrm{Fe} / \mathrm{H}]$, that is the opposite to what is seen for the $\alpha$-elements. While Nissen \& Schuster (2011) found that the high- $\alpha$ halo (enriched by Type Ia supernova) and low- $\alpha$ (not enriched) do not show different $[\mathrm{Mn} / \mathrm{Fe}]$, the amount of manganese depends on the mode of Type Ia supernova (Seitenzahl et al. 2013; Kobayashi et al. 2020b), and the dominant mode may be environment (metallicitiy) dependent (Kirby et al. 2019; de los Reyes et al. 2020).

As expected, therefore, Fig. 23 shows [Mn/Fe] increases linearly from $-0.3 \mathrm{dex}$ at $[\mathrm{Fe} / \mathrm{H}] \approx-1.0$, to approximately $0.2 \mathrm{dex}$ at $[\mathrm{Fe} / \mathrm{H}] \approx 0.5$; at lower metallicities there is a plateau with $[\mathrm{Mn} / \mathrm{Fe}] \approx-0.3$. This increasing trend is qualitatively consistent with what was found by Eitner et al. (2020), although their $[\mathrm{Mn} / \mathrm{Fe}]$ ratios are closer to solar at all metallicities. The difference may be because of neglected 3D effects, and we note that the Mn I $475.4 \mathrm{~nm}$ and $476.1 \mathrm{~nm}$ lines used in the present study appear to be sensitive to such effects (Fig. 17 of Bergemann et al. 2019). There is a much steeper trend of $[\mathrm{Mn} / \mathrm{Fe}]$ in LTE (Sect. 4.2). This LTE trend is consistent with previous LTE studies for dwarfs (Mishenina et al. 2015), and for giants from APOGEE (Hayes et al. 2018).

The K20 model predicts a steep trend of $[\mathrm{Mn} / \mathrm{Fe}]$ with $[\mathrm{Fe} / \mathrm{H}]$ at low metallicities, and an inflection at $[\mathrm{Fe} / \mathrm{H}] \approx-0.75$. The steep trend in the K20 model is in fact in better agreement with the LTE results than with the non-LTE results, at least at low metallicities. However, the inflection in the K20 model is not seen in either the LTE or non-LTE results, and may therefore be indicative of some missing physics.

\subsection{Barium}

Barium is a heavy element that is mainly produced in AGB stars, via the slow neutron capture process (Karakas \& Lattanzio 2014), and possibly also the intermediate neutron capture process (Hampel et al. 2016, 2019; Skúladóttir et al. 2020). In Fig. 24 the majority of stars sit at approximately $[\mathrm{Ba} / \mathrm{Fe}] \approx-0.1$ at $[\mathrm{Fe} / \mathrm{H}] \approx-1.0$, gradually rise to $[\mathrm{Ba} / \mathrm{Fe}] \approx 0.25$ at $[\mathrm{Fe} / \mathrm{H}] \approx$ -0.25 , and decrease again to $[\mathrm{Ba} / \mathrm{Fe}] \approx 0.0$ at $[\mathrm{Fe} / \mathrm{H}] \approx 0.25$. At lower metallicities the barium abundances are slightly elevated, at approximately $[\mathrm{Ba} / \mathrm{Fe}] \approx 0.5$ at $[\mathrm{Fe} / \mathrm{H}] \lesssim-1.0$.

The general behaviour of a peak at slightly sub-solar metallicities is in fact qualitatively similar to what was found in the LTE study of Delgado Mena et al. (2017), and in the non-LTE study of Korotin et al. (2011). In contrast, however, other LTE studies tend to find a flat trend in $[\mathrm{Ba} / \mathrm{Fe}]$, or one that very gradually increases with increasing $[\mathrm{Fe} / \mathrm{H}]$ (Mishenina et al. 2013; Bensby et al. 2014).

This decrease of $[\mathrm{Ba} / \mathrm{Fe}]$ with $[\mathrm{Fe} / \mathrm{H}]$ at the highest metallicities is not captured in the K20 model. It may suggest a lower production of barium from AGB stars at higher metallicities than what is found in the K20 model, such that iron pollution of Type Ia supernova dominates. It may also have a similar explanation to the trend for lithium (Sect. 5.1), namely that the highest metallicity stars are older stars that have migrated into the solar 
neighbourhood, since barium is sensitive to stellar age (Nissen 2016; Spina et al. 2018; Skúladóttir et al. 2019).

There is a large dispersion in Figs. 24 and 25. Some of this dispersion may be intrinsic. Studies of solar twins indicate that the youngest stars are enhanced in $[\mathrm{Ba} / \mathrm{Fe}]$ by approximately 0.2 dex (Fig. 4 of Nissen 2016). However, this interpretation cannot explain why the results for the giants appear to be skewed to higher barium abundances, as these are on average $1.5 \mathrm{Gyr}$ older than the dwarfs according to ages from GALAH DR3. As mentioned in Sect. 4.3, this offset may reflect other systematic errors within the GALAH analysis pipeline, not related to nonLTE effects (noting that the LTE trend is qualitatively similar to the non-LTE one). Possibly the problems are related to the choice of microturbulence, to which the Ba II lines are particularly sensitive (Dobrovolskas et al. 2012). The barium abundances presented here should therefore be treated with caution.

\section{Conclusion}

We have presented extensive grids of departure coefficients for 13 different elements, calculated on standard MARCS model atmospheres. These data can be found online (Amarsi 2020) or by contacting the lead author directly. The departure coefficients can be implemented into existing stellar analyses pipelines, to simply and cheaply account for departures from LTE for a large number of spectral lines.

We illustrated this latter point by implementing the grids into the GALAH analysis pipeline, that is based on the 1D spectrum synthesis code SME. The grids of departure coefficients clearly make an impact on large stellar surveys. The grids lead to differences in the mean $[\mathrm{A} / \mathrm{Fe}]$ at given $[\mathrm{Fe} / \mathrm{H}]$ of as much as 0.2 dex. This results in qualitatively different pictures of the Galactic chemical evolution, for example giving rise to metal-poor plateaus in $[\mathrm{Mg} / \mathrm{Fe}]$ (Fig. 18), [K/Fe] (Fig. 21), and [Mn/Fe] (Fig. 23), whereas a steep increase with increasing $[\mathrm{Fe} / \mathrm{H}]$ would be seen in LTE. The grids affect different parts of stellar parameter space differently, and thus for example remove offsets in $[\mathrm{Na} / \mathrm{Fe}]$ of approximately 0.2 dex between dwarfs and giants (Fig. 17). The grids generally reduce the dispersion in the $[\mathrm{A} / \mathrm{Fe}]$ versus $[\mathrm{Fe} / \mathrm{H}]$ plane (Fig. 25 ), by as much as $0.10 \mathrm{dex}$ in the case of [Si/Fe] (Fig. 20).

Future efforts will extend these calculations to more elements in the periodic table. This will make it possible to model even more elements in non-LTE, in GALAH as well as in other large spectroscopic surveys. This is a necessary step towards getting elemental abundances that are accurate to the 0.05 dex level, of the order of millions of stars.

We caution that there are nevertheless other systematics in stellar models that still need to be addressed. After taking departures from LTE into account, the next step may be to consider the effects of stellar convection (Nissen \& Gustafsson 2018). Unfortunately 3D non-LTE corrections (Amarsi et al. 2019b) cannot be implemented into existing stellar analyses pipelines in an analogous way to these pre-computed grids of 1D non-LTE departure coefficients; at present, grids of line-by-line 3D non-LTE abundance corrections are still required. However, in the more distant future, stellar analyses pipelines may move towards using 3D spectrum synthesis codes, and to using pre-computed grids of 3D non-LTE departure coefficients.

It is also unclear to what extent departures from LTE may impact the atmospheric stratifications themselves (Short \& Hauschildt 2005). The grids of departure coefficients presented here offer a way forward towards relaxing the LTE assumption in the construction of 1D model atmospheres. The departure coefficients for the key electron donors could be fed back into the model atmosphere code for a final iteration, to see the impact of departures from LTE on the atmospheric temperature stratification. This will be investigated in a future study.

Acknowledgements. The GALAH survey is based on observations made at the Anglo-Australia Telescope (AAT). We acknowledge the traditional owners of the land on which the AAT stands, the Gamilaraay people, and pay our respects to elders past and present. We thank Poul Erik Nissen for providing comments on the manuscript. A.M.A. and P.S.B. acknowledge support from the Swedish Research Council (VR 2016-03765), and the project grant "The New Milky Way" (KAW 2013.0052) from the Knut and Alice Wallenberg Foundation. M.A. gratefully acknowledges funding from the Australian Research Council (DP150100250 and FL110100012). H.R. acknowledges support from JHU. C.K. acknowledges funding from the UK Science and Technology Facility Council (STFC) through grants ST/M000958/1 and ST/R000905/1, and the Stromlo Distinguished Visitorship at the ANU. S.L.M. acknowledges support from the UNSW Scientia Fellowship program, and from the Australian Research Council (DP180101791). J.D.S. and D.B.Z. acknowledge support from the Australian Research Council (DP180101791). This work was also supported by the Australian Research Council Centre of Excellence for All Sky Astrophysics in 3 Dimensions (ASTRO 3D). Some of the computations were performed on resources provided (through projects SNIC 2018/3-465 and SNIC 2019/3-532) by the Swedish National Infrastructure for Computing (SNIC) at the Multidisciplinary Center for Advanced Computational Science (UPPMAX) and at the High Performance Computing Center North (HPC2N), partially funded by the Swedish Research Council through grant agreement no. 2016-07213. This work was supported by computational resources provided by the Australian Government through the National Computational Infrastructure (NCI) under the National Computational Merit Allocation Scheme.

\section{References}

Ahumada, R., Allende Prieto, C., Almeida, A., et al. 2020, ApJS, 249, 3

Allende Prieto, C., Asplund, M., \& Fabiani Bendicho P. 2004, A\&A, 423, 1109

Amarsi, A. M. 2020, http://doi .org/10.5281/zenodo. 3888393

Amarsi, A. M., \& Asplund, M. 2017, MNRAS, 464, 264

Amarsi, A. M., Asplund, M., Collet, R., \& Leenaarts, J. 2016a, MNRAS, 455, 3735

Amarsi, A. M., Lind, K., Asplund, M., Barklem, P. S., \& Collet, R. 2016b, MNRAS, 463, 1518

Amarsi, A. M., Barklem, P. S., Asplund, M., Collet, R., \& Zatsarinny, O. 2018a, A\&A, 616, A89

Amarsi, A. M., Nordlander, T., Barklem, P. S., et al. 2018b, A\&A, 615, A139

Amarsi, A. M., Barklem, P. S., Collet, R., Grevesse, N., \& Asplund, M. 2019a, A\&A, 624, A111

Amarsi, A. M., Nissen, P. E., \& Skúladóttir, Á. 2019b, A\&A, 630, A104

Amarsi, A. M., Grevesse, N., Grumer, J., et al. 2020, A\&A, 636, A120

Andrievsky, S. M., Spite, M., Korotin, S. A., et al. 2008, A\&A, 481, 481

Asplund, M. 2005, ARA\&A, 43, 481

Asplund, M., Grevesse, N., Sauval, A. J., \& Scott, P. 2009, ARA\&A, 47, 481

Barklem, P. S. 2016a, A\&ARv, 24, 9

Barklem, P. S. 2016b, Phys. Rev. A, 93, 042705

Barklem, P. S., \& Collet, R. 2016, A\&A, 588, A96

Barklem, P. S., Belyaev, A. K., \& Asplund, M. 2003, A\&A, 409, L1

Barklem, P. S., Belyaev, A. K., Dickinson, A. S., \& Gadéa, F. X. 2010, A\&A, 519, A20

Barklem, P. S., Belyaev, A. K., Guitou, M., et al. 2011, A\&A, 530, A94

Barklem, P. S., Belyaev, A. K., Spielfiedel, A., Guitou, M., \& Feautrier, N. 2012, A\&A, 541, A80

Bedell, M., Bean, J. L., Meléndez, J., et al. 2018, ApJ, 865, 68

Belyaev, A. K. 2013, Phys. Rev. A, 88, 052704

Belyaev, A. K., \& Barklem, P. S. 2003, Phys. Rev. A, 68, 062703

Belyaev, A. K., \& Yakovleva, S. A. 2017, A\&A, 606, A147

Belyaev, A. K., Barklem, P. S., Dickinson, A. S., \& Gadéa, F. X. 2010, Phys. Rev. A, 81, 032706

Belyaev, A. K., Barklem, P. S., Spielfiedel, A., et al. 2012, Phys. Rev. A, 85, 032704

Bensby, T., \& Lind, K. 2018, A\&A, 615, A151

Bensby, T., Feltzing, S., \& Oey, M. S. 2014, A\&A, 562, A71

Bergemann, M., Hansen, C. J., Bautista, M., \& Ruchti, G. 2012, A\&A, 546, A90

Bergemann, M., Collet, R., Amarsi, A. M., et al. 2017a, ApJ, 847, 15

Bergemann, M., Collet, R., Schönrich, R., et al. 2017b, ApJ, 847, 16

Bergemann, M., Gallagher, A. J., Eitner, P., et al. 2019, A\&A, 631, A80

Bonifacio, P., Spite, M., Cayrel, R., et al. 2009, A\&A, 501, 519 
Buder, S., Asplund, M., Duong, L., et al. 2018, MNRAS, 478, 4513

Chang, T. N., \& Tang, X. 1990, J. Quant. Spectr. Rad. Transf., 43, 207

Collet, R., Asplund, M., \& Trampedach, R. 2007, A\&A, 469, 687

Čotar, K., Zwitter, T., Kos, J., et al. 2019, MNRAS, 483, 3196

Dalton, G., Trager, S., Abrams, D. C., et al. 2016, Proc. SPIE, 9908, 99081G

Davidson, M. D., Snoek, L. C., Volten, H., \& Doenszelmann, A. 1992, A\&A, 255,457

de Jong, R. S., Agertz, O., Berbel, A. A., et al. 2019, The Messenger, 175, 3

de los Reyes, M. A. C., Kirby, E. N., Seitenzahl, I. R., \& Shen, K. J. 2020, ApJ, 891,85

De Silva, G. M., Freeman, K. C., Bland-Hawthorn, J., et al. 2015, MNRAS, 449, 2604

deBoer, R. J., Görres, J., Wiescher, M., et al. 2017, Rev. Mod. Phys., 89, 035007

Delgado Mena, E., Bertrán de Lis, S., Adibekyan, V. Z., et al. 2015, A\&A, 576, A69

Delgado Mena, E., Tsantaki, M., Adibekyan, V. Z., et al. 2017, A\&A, 606, A94

Den Hartog, E. A., Lawler, J. E., Sobeck, J. S., Sneden, C., \& Cowan, J. J. 2011, ApJS, 194, 35

Dobrovolskas, V., Kučinskas, A., Andrievsky, S. M., et al. 2012, A\&A, 540, A128

Drawin, H.-W. 1968, Z. Phys., 211, 404

Drawin, H. W. 1969, Z. Phys., 225, 483

Eitner, P., Bergemann, M., Hansen, C. J., et al. 2020, A\&A, 635, A38

Feuillet, D. K., Frankel, N., Lind, K., et al. 2019, MNRAS, 489, 1742

Fields, B. D. 2011, Ann. Rev. Nucl. Part. Sci., 61, 47

Fields, C. E., Timmes, F. X., Farmer, R., et al. 2018, ApJS, 234, 19

Froese Fischer, C., Tachiev, G., \& Irimia, A. 2006, At. Data Nucl. Data Tables, 92, 607

Gaia Collaboration (Brown, A. G. A., et al.) 2018, A\&A, 616, A1

Gallagher, A. J., Bergemann, M., Collet, R., et al. 2020, A\&A, 634, A55

Gao, X., Lind, K., Amarsi, A. M., et al. 2020, MNRAS, 497, L30

García Pérez, A. E., Allende Prieto, C., Holtzman, J. A., et al. 2016, AJ, 151, 144

Garz, T. 1973, A\&A, 26, 471

Grevesse, N., Asplund, M., \& Sauval, A. J. 2007, The Solar Chemical Composition, eds. R. von Steiger, G. Gloeckler, \& G. M. Mason (Berlin: Springer Science+Business Media), 105

Guiglion, G., Chiappini, C., Romano, D., et al. 2019, A\&A, 623, A99

Gustafsson, B., Edvardsson, B., Eriksson, K., et al. 2008, A\&A, 486, 951

Hampel, M., Stancliffe, R. J., Lugaro, M., \& Meyer, B. S. 2016, ApJ, 831, 171

Hampel, M., Karakas, A. I., Stancliffe, R. J., Meyer, B. S., \& Lugaro, M. 2019, ApJ, 887, 11

Hayden, M. R., Bovy, J., Holtzman, J. A., et al. 2015, ApJ, 808, 132

Hayes, C. R., Majewski, S. R., Shetrone, M., et al. 2018, ApJ, 852, 49

Hibbert, A., Biemont, E., Godefroid, M., \& Vaeck, N. 1991, J. Phys. B, 24 3943

Hibbert, A., Biemont, E., Godefroid, M., \& Vaeck, N. 1993, A\&AS, 99, 179

Hubeny, I., \& Mihalas, D. 2014, Theory of Stellar Atmospheres (Princeton, NJ: Princeton University Press )

Ibgui, L., Hubeny, I., Lanz, T., \& Stehlé, C. 2013, A\&A, 549, A126

Kao, C.-Y. J., \& Auer, L. H. 1990, Month. Weather Rev., 118, 1551

Karakas, A. I., \& Lattanzio, J. C. 2014, PASA, 31, e030

Karovicova, I., White, T. R., Nordlander, T., et al. 2018, MNRAS, 475, L81

Karovicova, I., White, T. R., Nordlander, T., et al. 2020, A\&A, 640, A25

Kaulakys, B. P. 1985, J. Phys. B, 18, L167

Kaulakys, B. P. 1986, JETP, 91, 391

Kaulakys, B. P. 1991, J. Phys. B, 24, L127

Kelleher, D. E., \& Podobedova, L. I. 2008, J. Phys. Chem. Ref. Data, 37, 709

Kirby, E. N., Xie, J. L., Guo, R., et al. 2019, ApJ, 881, 45

Kobayashi, C., Umeda, H., Nomoto, K., Tominaga, N., \& Ohkubo, T. 2006, ApJ, 653,1145

Kobayashi, C., Karakas, A. I., \& Lugaro, M. 2020a, ApJ, in press, [arXiv:2008.04660]

Kobayashi, C., Leung, S.-C., \& Nomoto, K. 2020b, ApJ, 895, 138

Korotin, S., Mishenina, T., Gorbaneva, T., \& Soubiran, C. 2011, MNRAS, 415, 2093

Korotin, S. A., Andrievsky, S. M., Hansen, C. J., et al. 2015, A\&A, 581, A70

Kos, J., Lin, J., Zwitter, T., et al. 2017, MNRAS, 464, 1259

Kramida, A., Ralchenko, Y., Reader, J., et al. 2012, NIST atomic spectra database (version 5)

Kroupa, P. 2008, ASP Conf. Ser., 390, 3

Lambert, D. L. 1993, Phys. Scr. T, 47, 186

Lee, H.-W., \& Kim, H. I. 2004, MNRAS, 347, 802

Leenaarts, J., \& Carlsson, M. 2009, ASP Conf. Ser., 415, 87
Lind, K., Asplund, M., Barklem, P. S., \& Belyaev, A. K. 2011, A\&A, 528, A103

Lind, K., Bergemann, M., \& Asplund, M. 2012, MNRAS, 427, 50

Lind, K., Melendez, J., Asplund, M., Collet, R., \& Magic, Z. 2013, A\&A, 554, A96

Lind, K., Amarsi, A. M., Asplund, M., et al. 2017, MNRAS, 468, 4311

Liu, C., Fu, J., Shi, J., et al. 2020, Res. Astron. Astrophys., submitted [arXiv:2005.07210]

Martell, S., Simpson, J., Balasubramaniam, A., et al. 2020, MNRAS, submitted [arXiv:2006.02106]

Mashonkina, L. I., Sitnova, T. N., \& Pakhomov, Y. V. 2016, Astron. Lett., 42, 606

Mashonkina, L. I., Neretina, M. D., Sitnova, T. M., \& Pakhomov, Y. V. 2019 Astron. Rep., 63, 726

McWilliam, A. 1997, ARA\&A, 35, 503

Mihalas, D., \& Athay, R. G. 1973, ARA\&A, 11, 187

Mishenina, T. V., Pignatari, M., Korotin, S. A., et al. 2013, A\&A, 552, A128

Mishenina, T., Gorbaneva, T., Pignatari, M., Thielemann, F. K., \& Korotin, S. A. 2015, MNRAS, 454, 1585

Müller, B. 2016, PASA, 33, e048

Ness, M., Hogg, D. W., Rix, H.-W., Ho, A. Y. Q., \& Zasowski, G. 2015, ApJ, 808,16

Nissen, P. E. 2015, A\&A, 579, A52

Nissen, P. E. 2016, A\&A, 593, A65

Nissen, P. E., \& Gustafsson, B. 2018, A\&ARv, 26, 6

Nissen, P. E., \& Schuster, W. J. 2010, A\&A, 511, L10

Nissen, P. E., \& Schuster, W. J. 2011, A\&A, 530, A15

Nordlander, T., \& Lind, K. 2017, A\&A, 607, A75

O’Brian, T. R., \& Lawler, J. E. 1991, Phys. Rev. A, 44, 7134

Osorio, Y., \& Barklem, P. S. 2016, A\&A, 586, A120

Osorio, Y., Barklem, P. S., Lind, K., et al. 2015, A\&A, 579, A53

Osorio, Y., Lind, K., Barklem, P. S., Allende Prieto, C., \& Zatsarinny, O. 2019, A\&A, 623, A103

Osorio, Y., Allende Prieto, C., Hubeny, I., Mészáros, S., \& Shetrone, M. 2020, A\&A, 637, A80

Pinsonneault, M. 1997, ARA\&A, 35, 557

Piskunov, N., \& Valenti, J. A. 2017, A\&A, 597, A16

Prantzos, N. 2012, A\&A, 542, A67

Ramírez, I., Meléndez, J., \& Chanamé, J. 2012, ApJ, 757, 164

Randich, S., Gilmore, G., \& Gaia-ESO Consortium. 2013, The Messenger, 154, 47

Recio-Blanco, A., de Laverny, P., Allende Prieto, C., et al. 2016, A\&A, 585, A93

Reggiani, H., Amarsi, A. M., Lind, K., et al. 2019, A\&A, 627, A177

Romano, D., Matteucci, F., Zhang, Z.-Y., Ivison, R. J., \& Ventura, P. 2019, MNRAS, 490, 2838

Romano, D., Franchini, M., Grisoni, V., et al. 2020, A\&A, 639, A37

Rutten, R. J. 2003, Radiative Transfer in Stellar Atmospheres, 8th edn. (Utrecht: Utrecht University)

Rybicki, G. B., \& Hummer, D. G. 1992, A\&A, 262, 209

Saad, Y. 2003, Iterative methods for sparse linear systems, Vol. 82 (SIAM)

Seitenzahl, I. R., Cescutti, G., Röpke, F. K., Ruiter, A. J., \& Pakmor, R. 2013, A\&A, 559, L5

Short, C. I., \& Hauschildt, P. H. 2005, ApJ, 618, 926

Silva Aguirre, V., Casagrande, L., Basu, S., et al. 2012, ApJ, 757, 99

Skúladóttir, Á., Hansen, C. J., Salvadori, S., \& Choplin, A. 2019, A\&A, 631, A 171

Skúladóttir, Á., Hansen, C. J., Choplin, A., et al. 2020, A\&A, 634, A84

Smiljanic, R., Romano, D., Bragaglia, A., et al. 2016, A\&A, 589, A115

Smith, G. 1988, J. Phys. B, 21, 2827

Smith, G., \& Raggett, D. S. J. 1981, J. Phys. B, 14, 4015

Spina, L., Meléndez, J., \& Ramírez, I. 2016, A\&A, 585, A152

Spina, L., Meléndez, J., Karakas, A. I., et al. 2018, MNRAS, 474, 2580

Spite, F., \& Spite, M. 1982, A\&A, 115, 357

Steenbock, W., \& Holweger, H. 1984, A\&A, 130, 319

Steffen, M., Prakapavičius, D., Caffau, E., et al. 2015, A\&A, 583, A57

Steinmetz, M., Guiglion, G., McMillan, P. J., et al. 2020, AJ, 160, 83

Stonkute, E., Chorniy, Y., Tautvaišienè, G., et al. 2020, AJ, 159, 90

Thomson, J. J. 1912, Lond. Edin. Dublin Philos. Mag. J. Sci., 23, 449

Ting, Y.-S., Conroy, C., Rix, H.-W., \& Cargile, P. 2019, ApJ, 879, 69

Trubko, R., Gregoire, M. D., Holmgren, W. F., \& Cronin, A. D. 2017, Phys. Rev. A, 95, 052507

Valenti, J. A., \& Piskunov, N. 1996, A\&AS, 118, 595

van Regemorter H. 1962, ApJ, 136, 906

Xiang, M., Ting, Y.-S., Rix, H.-W., et al. 2019, ApJS, 245, 34

Yan, Z.-C., Tambasco, M., \& Drake, G. W. F. 1998, Phys. Rev. A, 57, 1652

Zhao, G., Mashonkina, L., Yan, H. L., et al. 2016, ApJ, 833, 225 